\title{
An inverse eigenvalue problem with rotational symmetry
}

\author{
Thomas I Seidman $\dagger$ \\ Department of Mathematics, University of Maryland, Baltimore County, Baltimore, MD \\ 21228, USA
}

Received 3 August 1987, in final form 12 February 1988

\begin{abstract}
We consider convergence of an approximation method for the recovery of a rotationally symmetric potential $\psi$ from the sequence of eigenvalues. In order to permit the consideration of 'rough' potentials $\psi$ (having essentially $H^{-1}(0,1)$ regularity), we first indicate the appropriate interpretation of $-\Delta+\psi$ (with boundary conditions) as a self-adjoint, densely defined operator on $\mathscr{H}:=L^{2}(\Omega)$ and then show a suitable continuous dependence on $\psi$ for the relevant eigenvalues. The approach to the inverse problem is by the method of 'generalised interpolation' and, assuming uniqueness, it is shown that one has convergence to the correct potential $\psi$ (strongly, for an appropriate norm) for a sequence of computationally implementable approximations $\left(\boldsymbol{P}_{\mathrm{c}, N}\right)$.
\end{abstract}

\section{Introduction}

The present paper is intended as an extension of the considerations of [1] to higherdimensional contexts. Our concern will be with formal operators

$$
\boldsymbol{L}=\boldsymbol{L}_{\psi}: u \rightarrow-\nabla \cdot a \nabla u+\psi u
$$

in a context of rotational symmetry in $\mathbb{R}^{d}$, i.e. assuming that

(i) $\quad a(\cdot), \psi(\cdot)$ depend only on $r:=|x|$,

(ii) the domain $\Omega$ is the unit ball of $\mathbb{R}^{d}$ with $d \geqslant 2$,

(iii) the boundary conditions are radial, of the form

$$
a u_{r}=\gamma u \text { on } \partial \Omega \text { (i.e. at } r=1 \text { ). }
$$

We assume $a(\cdot)$ is known and bounded with a uniform ellipticity condition:

$$
A \geqslant a(r) \geqslant \alpha>0 \quad \text { for } 0 \leqslant r \leqslant 1
$$

(e.g. $a \equiv 1$ giving $L_{0}=-\Delta$ ).

Our concern is with the inverse eigenvalue problem (EVP):

suppose $a(\cdot)$ is known and it is known that $\psi \in \Psi_{*}$ (some suitable set); if we are given eigenvalues of the self-adjoint operator $\boldsymbol{A}_{\psi}$ associated with (1.1) and (1.2) (iii), how can we (computationally) recover the potential $\psi$ ?

† Electronic address on BITNET: seidman @ umbc.

$\ddagger$ We could equally well consider Dirichlet conditions $(u=0$ at $r=1)$, which would require minor modification of our presentation, e.g. we would set $\mathscr{V}:=H_{0}^{1}(\Omega)$ rather than $H^{\mathrm{t}}(\Omega)$ as here, etc. 
We do not consider here the deep question of uniqueness: within which sets $\Psi_{*}$ is $\psi$ uniquely determined by the given eigenvalue information? Rather, this is taken as an a priori assumption on the suitability of $\psi_{*}$ for EVP.

On the other hand, as in [1], we are very much concerned with another aspect of the suitability of $\Psi_{*}$ : for how 'rough' a potential $\psi$ can we construct a workable interpretation of $(-\nabla \cdot a \nabla+\psi)$ as a densely defined, self-adjoint operator $\boldsymbol{A}_{\psi}$ on $L^{2}(\Omega)$ with compact resolvent so that discussion of the 'eigenvalues of . . $\boldsymbol{A}_{\psi}$ ' makes sense? The approach, as in [1], is closely related to that of chapter 3 of [2] with modification to fit the setting under consideration. In [1] it was shown, for the onedimensional case, that $\boldsymbol{A}_{\psi}$ is suitably defined for $\psi \in \mathscr{P}^{*}$ with $\mathscr{P}:=H^{1}(-1,1)$ and that the eigenvalues then depend continuouslya on $\psi$. A principal concern here will be to obtain comparable results for $\psi \in \mathscr{P}^{*}$ with $\mathscr{P}$ much like $H^{1}(0,1)$-viewing $\psi=\psi(r)$ as given for $r \in(0,1)$, rather than on $\Omega$-but now with $\mathscr{P}$ defined through a weighted $H^{1}$ norm, controlling the behaviour near $r=0$. We are able to get results quite comparable with the one-dimensional case treated in [1] precisely because the radial symmetry permits a treatment through separation of variables which reduces this to one-dimensional considerations.

Once we have developed the setting in which EvP is a meaningful problem, our concern is to demonstrate convergence for an approximation method of 'generalised interpolation' type (see, e.g., [2, 3]). We assume in EVP that we are given the sequence $\left(\bar{\lambda}_{1}, \bar{\lambda}_{2}, \ldots\right)$ of the eigenvalues of $\boldsymbol{A}_{\bar{\psi}}$ corresponding to a unique potential $\bar{\psi} \in \Psi_{*}$ and consider the approximation procedure:

$$
\begin{aligned}
& \left(\boldsymbol{P}_{N}\right) \quad \text { Let } \Psi_{N}:=\left\{\psi \in \Psi_{*}: \lambda_{k}(\psi)=\bar{\lambda}_{k} \text { for } k=1, \ldots, N\right\} \\
& \quad \text { and select } \psi_{N} \in \Psi_{N} \text { to minimise }\|\psi\|_{*} \text { over } \Psi_{N} \text { for } N=1,2, \ldots
\end{aligned}
$$

The particular norm used for minimisation in $\left(\boldsymbol{P}_{N}\right)$ must be appropriately related to the continuity of the functionals $\lambda_{j}(\cdot)$ and we then expect strong convergence:

$$
\psi_{N} \rightarrow \bar{\psi} \quad \text { in the sense of }\|\cdot\|_{*} \text { as } N \rightarrow \infty .
$$

We will demonstrate convergence for a modification of $\left(\boldsymbol{P}_{N}\right)$, weakened to permit the use of computational approximations to the functionals $\lambda_{k}(\cdot)$ and an approximate minimisation for the norm.

\section{The operator}

We are concerned in this section to define a self-adjoint (closed, unbounded, densely defined) operator

$$
\boldsymbol{A}_{\psi}: \mathscr{H} \supset \mathscr{D}_{\psi} \rightarrow \mathscr{H}
$$

on $\mathscr{H}:=L^{2}(\Omega)$ with compact resolvent corresponding to the formal operator $\boldsymbol{L}_{\psi}$. We note three definitions/interpretations of increasing generality. topology for $\psi$ rather than the sequential weak topology as asserted there. See theorem 11 below. 
(i) For $a, \psi, u$ 'smooth enough' one has the classical interpretation of $\boldsymbol{L}_{\psi}$, computing pointwise in (1.1) and (1.2) (iii). For smooth $a, \psi$, one still has such a pointwise interpretation of (1.1) and, via trace theory, of (1.2) (iii), for $u \in H^{2}(\Omega)$, so we can take $\mathscr{D}_{\psi}^{(\mathrm{i})}:=\left\{u \in H^{2}(\Omega): a u_{r}=\gamma u\right.$ on $\left.\partial \Omega\right\}$.

(ii) For $a \in L^{\infty}$ and $\psi$ in a certain $L^{\bar{q}}(\Omega)$ (see below) we have a 'weak interpretation' of $\left(\boldsymbol{L}_{\psi}+\lambda\right)$ as a continuous invertible operator: $\mathscr{V} \rightarrow \mathscr{V}^{*}$ with $\mathscr{V}=H^{1}(\Omega)$. We can then take $\mathscr{D}_{\psi}^{(i i)}$ to be the pre-image of $\mathscr{H} \subset \mathscr{V}^{*}$ for this operator.

(iii) The radial nature of $\boldsymbol{L}_{\psi}$ induces (e.g. for smooth $a, \psi$ ) a canonical decomposition of $\mathscr{H}$ by separation of variables into subspaces of the form $\mathscr{H}_{\mu}=\mathscr{X} \otimes \mathcal{U}_{\mu}$ where each $u_{\mu}$ is finite dimensional $\dagger$ and $\mathscr{X}$ is a weighted $L^{2}$ space of functions on $(0,1)$. Associated with $-\nabla \cdot a \nabla \cdot$ and (1.2) (iii) is an ordinary differential operator $\boldsymbol{M}_{\mu}$ and, following [4], we can interpret $\left(\boldsymbol{M}_{\mu}+\psi\right)$ as a self-adjoint operator on $\mathscr{X}$ for each relevant $\mu$ when $\psi$ is in $\mathscr{P}^{*}$ where, now, $\mathscr{P}$ is a weighted $H^{1}$ space on $(0,1)$. These interpretations $\boldsymbol{A}_{\mu, \psi}: \mathscr{H}_{\mu} \supset \mathscr{D}_{\mu, \psi} \rightarrow \mathscr{H}_{\mu}$ can be combined to obtain $\mathscr{D}_{\psi}=\mathscr{D}_{\psi}^{\text {(iii) }}$ and the interpretation of (2.1).

We will ultimately use the interpretation (iii) but, of course, wish to know that the interpretations are consistent with each other.

For a more unified treatment of the two interpretations (ii), (iii) we proceed, for the moment, in a somewhat abstract fashion. For (ii) we take $\mathscr{X}=\mathscr{H}\left(=L^{2}(\mathscr{P})\right.$ with $\mathscr{S}=\Omega), \mathscr{Y}=\mathscr{V}$ and observe that $\boldsymbol{L}_{0}$ induces, in an obvious way, a continuous operator $\boldsymbol{M}: \mathscr{Y} \rightarrow \mathscr{Y}^{*}$. Following [1] we construct the operator $\boldsymbol{A}_{\psi}$ on $\mathscr{X}=\mathscr{H}$ from this operator $\boldsymbol{M}$ and the multiplication operator: $\mathscr{Y} \rightarrow \mathcal{Y}^{*}$ induced by $\psi$. For (iii) we use the 'separation of variables' decomposition $\mathscr{H}=\oplus \mathscr{H}_{\mu}$ to work with spaces of functions of $r \in \mathscr{S}=(0$, 1)-for each $\mu \in \sigma(S)$ we take $\mathscr{Q}$ to be a suitably weighted $L^{2}(\mathscr{S})$ and $\mathscr{Y}$ to be a suitably weighted $H^{1}(\mathscr{S})$. Again we will have bounded linear operators $\boldsymbol{M}=\boldsymbol{M}_{\mu}$ and multiplication by $\psi$ acting: $y \rightarrow y^{*}$. The separate pieces, each obtained by the abstract procedure following [1], can then be put together to provide the interpretation (iii) of $\boldsymbol{A}_{\psi}$.

In each case $\mathscr{X}$ is of the form $L_{\rho}^{2}(\mathscr{Y})(\mathscr{Y}=\Omega$ for (ii); $\mathscr{S}=(0,1)$ for (iii) with $\rho$ a positive bounded measure on $\mathscr{Y}$ ) and $\mathscr{Y}$ is also a Hilbert space of functions on $\mathscr{S}$ with a pivoting

$$
y \hookrightarrow x \hookrightarrow y^{*}
$$

with dense embeddings. In each case we have a linear continuous map $\boldsymbol{M}: y \rightarrow y^{*}$ for which one has a monotonicity estimate of the form

$$
\langle\boldsymbol{M} x, x\rangle \geqslant \underline{\alpha}|x|_{\mathscr{Y}}^{2}-\beta|x|_{\mathscr{X}}^{2} \quad x \in \mathcal{Y} \subset \mathscr{X}
$$

with $\underline{\alpha}>0$. We will also have symmetry:

$$
\langle\boldsymbol{M x}, y\rangle=\langle x, \boldsymbol{M} y\rangle \quad \text { for } x, y \in \mathcal{y} .
$$

(The $9-9$ dualities of $^{*}$ (2.3) and (2.4) are, of course, given by the pivoting through the inner product of $\mathscr{X}$.)

† The elements of $~$ are just the classical 'angular' functions well known from analysis of the Laplacian for a ball, i.e. $\{\sin n \theta, \cos n \theta\}$ for $d=2$, spherical harmonics for $d=3$, etc. The subspaces $\left\{\cup_{\mu}\right\}$ do not depend on $a, \psi$ or the boundary conditions and give an orthogonal direct sum decomposition of $L^{2}(\partial \Omega)$. 
Next, take $\mathscr{P}$ to be any space containing products $x y$ for $x, y \in \mathcal{Y}$ with a norm such that $\dagger$

$$
|x y|_{\mathscr{P}} \leqslant \bar{C}|x|_{\mathscr{O}}|y|_{\mathscr{O}} \quad \text { for } x, y \in \mathscr{Y}
$$

Lemma 1. Let $\psi$ be a function on $\mathscr{Y}$ which is in $\mathscr{P}^{*}$ in the sense of the $\mathscr{P}-\mathscr{P}^{*}$ duality induced by the $\mathscr{X}$ inner product. Then the multiplication operator

$$
\psi: x \mapsto \psi x: \text { oy } \rightarrow y^{*}
$$

is well defined and continuous with

$$
|\psi x|_{a^{*}} \leqslant \tilde{C}|\psi|_{\mathscr{P}^{*}}|x|_{y y} \quad \text { so } \quad\|\psi\| \leqslant \tilde{C}|\psi|_{\mathscr{P}^{*}}
$$

Proof. From (2.5) we have

$$
|\langle\psi x, y\rangle|=|\langle\psi, x y\rangle| \leqslant|\psi|_{\mathscr{P}^{*}}|x y|_{\mathscr{P}} \leqslant\left(\bar{C}|\psi|_{\mathscr{P}^{*}}|x|_{y y}\right)|y|_{\dddot{y}}
$$

for arbitrary $x, y \in \mathscr{Y}$. By the definition of the $y^{*}$ norm as $\sup \left\{|\langle\psi x, y\rangle|:|y|_{y} \leqslant 1\right\}$, this gives (2.6).

Note that for such functions $\psi, \varphi$ we have $\varphi \geqslant \psi$ precisely when $\langle(\psi-\varphi) y, y\rangle$ $=\left\langle\psi-\varphi, y^{2}\right\rangle \geqslant 0$ for $y \in \mathscr{Y}$ and we take this as inducing the order for $\mathscr{P}^{*}$. We also wish to consider $\varphi \in \mathscr{P}^{*}$ (so that $\varphi: \mathscr{Y} \rightarrow \mathscr{Y}^{*}$ is defined) such that for each $\varepsilon>0$ one has $C_{\varepsilon}$ such that

$$
|\langle\varphi x, x\rangle| \leqslant \varepsilon|x|_{\mathscr{O}}^{2}+C_{\varepsilon}|x|_{\mathscr{X}}^{2} \quad \text { for } x \in \mathscr{Y} \subset \mathscr{X} .
$$

Lemma 2. Let $\mathscr{X}, \mathscr{Y}, \mathscr{P}$ be as above. Let $\boldsymbol{M}: \mathscr{Y} \rightarrow \mathscr{Y}^{*}$, as above, satisfy $(2.3),(2.4)$ and let $\psi \in \mathscr{P P}^{*}$ with $\psi \geqslant \varphi$ for $\varphi$ satisfying (2.7). Then $(\boldsymbol{M}+\psi)$ induces a densely defined, self-adjoint operator $\boldsymbol{M}_{\psi}: \mathscr{X} \supset \mathscr{D}_{\psi} \rightarrow \mathscr{X}$. If the embedding $\mathscr{Y} \hookrightarrow \mathscr{X}$ induced by the pivoting is compact, then $\boldsymbol{M}_{\psi}$ has compact resolvent.

Proof. For any real $\lambda$ we have $(\boldsymbol{M}+\psi+\lambda): y \rightarrow y^{*}$ continuous with

$$
\begin{aligned}
\langle(\boldsymbol{M}+\psi+\lambda) x, x\rangle & =\langle\boldsymbol{M} x, x\rangle+\langle\lambda+\varphi) x, x\rangle+\left\langle\psi-\varphi, x^{2}\right\rangle \\
& \geqslant\left[\underline{\alpha}|x|_{\mathscr{y}}^{2}-\beta|x|_{\mathscr{2}}^{2}\right]+\lambda|x|_{\mathscr{x}}^{2}+\langle\varphi x, x\rangle
\end{aligned}
$$

using (2.3) and noting $\psi \geqslant \varphi$. Using an inequality $|x|_{\mathscr{E}} \leqslant c_{0}|x|_{y}$ and (2.7) with $\varepsilon:=\underline{\alpha} / 2 c_{0}$, we obtain the fundamental estimate

$$
\langle(M+\psi+\lambda) x, x\rangle \geqslant(\underline{\alpha} / 2)|x|_{\mathscr{y}}^{2}+\left(\lambda-\beta-c_{0} C_{\varepsilon}\right)|x|_{\mathscr{x}}^{2} .
$$

Considering $\lambda \geqslant \beta+c_{0} C_{\varepsilon}=: \bar{\lambda}_{\varphi}$, this makes $(M+\psi+\lambda): \bigcirc \rightarrow \circlearrowleft *$ strictly monotonic and hence invertible. We set

$$
\begin{aligned}
\mathscr{D}_{\psi} & :=\mathscr{R}\left(\left.(\boldsymbol{M}+\psi+\lambda)^{-1}\right|_{\mathscr{X}}\right) \\
& =\{x \in \mathscr{Y}:(\boldsymbol{M}+\psi+\lambda) x=: z \in \mathscr{X}\} \subset \mathscr{X} \\
\boldsymbol{M}_{\psi} x & :=z-\lambda x \text { for } x \in \mathscr{D}_{\psi} \text { with } z:=(\boldsymbol{M}+\psi+\lambda) x \in \mathscr{X} .
\end{aligned}
$$

$\dagger$ We remark that if there is any $\mathscr{P}$ norm giving (2.5), then

$$
u \mapsto \inf \left\{\sum_{j}\left|x_{j} \| y_{j}\right|: \sum_{j} x_{j} y_{j}=u ;|\cdot|=|\cdot| y\right\}
$$

is a norm on $\operatorname{sp}\{x y: x, y \in \mathscr{Y}\}$ and canonically defines the (essentially unique) strongest norm topology giving (2.5). 
The continuity of $(\boldsymbol{M}+\psi+\lambda)^{-1}: \mathscr{X} \hookrightarrow \mathscr{Y}^{*} \rightarrow \mathscr{Y}$ ensures that $\boldsymbol{A}_{\psi}$ is a closed operator. Clearly, this definition is independent of the particular choice of (large enough) $\lambda$. Note that $\tilde{\lambda}_{\varphi}$ depends on $\psi$ only through the lower bound $\varphi$ but (2.9) does not depend on the particular choice of $\varphi$. Fixing $\varphi$ and taking $\lambda \geqslant \bar{\lambda},(2.8)$ gives

$$
|x|_{\mathscr{y}} \leqslant M\left|\left(\boldsymbol{M}_{\psi}+\lambda\right)\right|_{\mathscr{X}} \quad \text { for } x \in \mathscr{D}_{\psi}
$$

uniformly on $\left\{\psi \in \mathscr{P}^{*}: \psi \geqslant \varphi\right\}$ with $M=2 / \underline{\alpha} \bar{C}$ where $\bar{C}:=$ [norm of the embedding: $\mathscr{Y} \rightarrow \mathscr{X}]$. Note that if $\mathscr{Y} \rightarrow \mathscr{X}$ is compact then $(2.10)$ makes $\left\{\left(\boldsymbol{M}_{\psi}+\lambda\right)^{-1}: \lambda \geqslant \bar{\lambda}_{\varphi}, \psi \geqslant \varphi\right.$, $\left.\psi \in \mathscr{P}^{*}\right\}$ collectively compact for any $\varphi$ satisfying (2.7).

To see that $\mathscr{D}_{\psi}$ is dense in $\mathscr{X}$, i.e. that $(\boldsymbol{M}+\psi+\lambda)^{-1}: \mathscr{X} \rightarrow \mathscr{X}$ has dense range, we proceed by contradiction. Were $\mathscr{D}_{\psi}$ not dense there would exist $\bar{x} \in \mathscr{X}$ orthogonal to $\mathscr{D}_{\psi}$ with $\bar{x} \neq 0$. We could then find $\bar{z} \in \mathcal{Y}$ with $(M+\psi+\lambda) \bar{z}=\bar{x}$ so, using (2.4)

$$
\begin{aligned}
\langle\bar{z}, z\rangle & =\left\langle(\boldsymbol{M}+\psi+\lambda) \bar{z},(\boldsymbol{M}+\psi+\lambda)^{-1} z\right\rangle \\
& =\left\langle\bar{x},(\boldsymbol{M}+\psi+\lambda)^{-1} z\right\rangle=0 \quad \text { since }(\boldsymbol{M}+\psi+\lambda)^{-1} z \in \mathscr{D}_{\psi}
\end{aligned}
$$

for any $z \in \mathscr{X}$. Hence $\bar{z}=0$ so $\bar{x}=0$-a contradiction.

Finally, the assumed symmetry of $\boldsymbol{M}$ makes $\boldsymbol{M}_{\psi}$ formally self-adjoint but we must verify that the domain of $\left(\boldsymbol{M}_{\psi}\right)^{*}$ is precisely $\mathscr{D}_{\psi}$, i.e. that $\mathscr{Z}$-continuity (on the dense set $\mathscr{D}_{\psi}$ ) of the functional $y \rightarrow\left\langle\boldsymbol{M}_{\psi} y, x\right\rangle$ implies $x \in \mathscr{D}_{\psi}$ (noting that the inverse implication is clear). This continuity implies existence of $z \in \mathscr{X}$ such that $\left\langle\boldsymbol{M}_{\psi} y, x\right\rangle=\langle y, z\rangle$ for each $y \in \mathscr{D}_{\psi}$; set

$$
\tilde{x}:=(M+\psi+\lambda)^{-1}[z+\lambda x] \in \mathscr{D}_{\psi} .
$$

We then have, by the symmetry,

$$
\begin{aligned}
\langle(\boldsymbol{M}+\psi+\lambda) y, \tilde{x}\rangle & =\langle\boldsymbol{M} y, \tilde{\boldsymbol{x}}\rangle+\langle(\psi+\lambda) y, \tilde{x}\rangle \\
& =\langle y, \boldsymbol{M} \tilde{x}\rangle+\langle y,(\psi+\lambda) \tilde{x}\rangle \\
& =\langle y,(\boldsymbol{M}+\psi+\lambda) \tilde{x}\rangle-\langle y, z+\lambda x\rangle \\
& =\left\langle\boldsymbol{M}_{\psi} y, x\right\rangle+\lambda\langle y, x\rangle \\
& =\langle(\boldsymbol{M}+\psi+\lambda) y, x\rangle .
\end{aligned}
$$

Since $(M+\psi+\lambda) y$ ranges over $\mathscr{X}$ as $y$ ranges over $\mathscr{D}_{\psi}$, this shows $\tilde{x}=x$ so $x \in \mathscr{D}_{\psi}$. 
For the interpretation (2.2) (ii) of $\boldsymbol{L}_{\psi}$, we take $\dagger \mathscr{y}=\mathscr{V}:=H^{1}(\Omega)$ and $\mathscr{X}=\mathscr{H}:=L^{2}(\Omega)$. The standard weak formulation of $A:=-\nabla \cdot a \nabla \cdot$ with (1.2) (iii) is given by

$$
\begin{aligned}
\langle\boldsymbol{A} u, v\rangle & =\int_{\Omega}(-\nabla \cdot a \nabla u) v \\
& =\int_{\Omega} a \nabla u \cdot \nabla v-\gamma \int_{\partial \Omega} u v .
\end{aligned}
$$

Since [5] the Dirichlet trace is compact: $y \rightarrow L^{2}(\partial \Omega)$, we have

$$
\left|\int_{\partial \Omega} u v\right| \leqslant \varepsilon|u|_{V}|v|_{\mathscr{V}}+C_{\varepsilon}|u|_{\mathscr{H}}|v|_{\mathscr{H}} \quad \text { for } u, v \in \mathcal{V}
$$

by a 'standard' functional analysis result $\ddagger$. Hence, taking

$$
|u|_{\mathcal{V}}:=\left(\int_{\Omega}|\nabla u|^{2}+|u|^{2}\right)^{1 / 2}
$$

we obtain (2.3) for $\boldsymbol{M}=\boldsymbol{A}$ with $\underline{\alpha}$ arbitrarily close to the $\alpha$ in (1.3) and a correspondingly determined $\beta$. We now take $\S \bar{q}:=2 d /(d+2)>1$ so that (with $1 / \bar{q}+1 / \bar{p}=1$ ) standard results [5] give continuous embedding: $\mathscr{V} \rightarrow L^{2 \tilde{p}}(\Omega)$ whence

$$
\left|x^{2}\right|_{\bar{p}}=|x|_{2 \bar{p}}^{2} \leqslant C|x|_{\mathfrak{r}}^{2} .
$$

This means that we can take $\mathscr{P}=L^{\bar{p}}(\Omega)$ and $\psi \in \mathscr{P}^{*}=L^{\bar{q}}(\Omega)$. For $q>\bar{q}$ (or $q=\bar{q}$ when $d=2$ ) the embedding: $\mathscr{V} \rightarrow L^{2 p}(\Omega)$ (with $1 / q+1 / p=1$ ) is compact so we have $(2.7)$, for $\varphi \in L^{q}(\Omega)$. We have thus shown the following.

For any $\psi \in L^{\bar{q}}(\Omega)$, i.e. $\int r^{d-1}|\psi(r)\rangle^{\bar{q}} \mathrm{~d} r<\infty$, bounded below by $\varphi \in L^{q}(\Omega)$, the construcstruction (2.2) (ii) via (2.9) defines $\boldsymbol{A}_{\psi}$, corresponding to $\boldsymbol{L}_{\psi}$ in (1.1), as a densely defined, self-adjoint operator on $L^{2}(\Omega)$ with compact resolvent.

When $a$ and $\psi$ are smooth it is a standard regularity result that $u \in H^{2}(\Omega)$ for $\boldsymbol{L}_{\psi} u \in L^{2}(\Omega)$ so the definitions (2.2) (i), (ii) are then equivalent.

We proceed now to develop (2.2) (iii). A formal calculation\|, imposing the ansatz

$$
u(x)=R(r) U(\omega) \quad \text { for } x=r \omega \in \Omega
$$

with $r \in(0,1)$ and $\omega \in S^{d-1}=\partial \Omega$, gives

$$
-\nabla \cdot a \nabla u=\left(M_{0} R\right) U+\frac{a}{r^{2}} R(S U)
$$

$\dagger$ As noted earlier, we have $\mathscr{V}:=H_{0,}^{1}(\Omega)$ in the case of Dirichlet boundary conditions.

$\ddagger$ Theorem. Let $\mathscr{U}, \mathcal{V}, \mathcal{W}$ be Banach spaces with $\mathscr{V}$ reflexive and suppose $A: \mathscr{V} \rightarrow \mathcal{U}$ and $\boldsymbol{B}: \mathscr{V} \rightarrow \mathscr{W}$ are continuous linear maps with $\boldsymbol{A}$ compact and $\mathcal{N}(\boldsymbol{B}) \subset \mathcal{N}(\boldsymbol{A})$ (i.e. $\boldsymbol{B} v=0 \Rightarrow \boldsymbol{A} v=0$ ). Then for each $\varepsilon>0$ there exists $C=C_{\varepsilon}$ such that $|\boldsymbol{A} v|_{\mathscr{q} \ell} \leqslant \varepsilon|v|_{\mathcal{V}}+C_{\varepsilon}|\boldsymbol{B} v|_{\mathcal{W}}$ for $v \in \mathcal{V}$.

Proof. Suppose not. Then there would exist $\varepsilon_{*}>0$ and $\left\{v_{k}\right\}$ in $\mathscr{V}$ with $\left|v_{k}\right|_{V}=1,\left|\boldsymbol{A} v_{k}\right|_{u} \geqslant \varepsilon_{*}\left|v_{k}\right|_{Y}+k\left|\boldsymbol{B} v_{k}\right|_{w}$. Extract a subsequence so $v_{k} \rightarrow v_{*}$ (whence $\boldsymbol{B} v_{k} \rightarrow \boldsymbol{B} v_{*}$ ) and $\boldsymbol{A} v_{k} \rightarrow \boldsymbol{A} v_{*}$, using the reflexivity of $\mathcal{V}$ and the compactness of $\boldsymbol{A}$. One has $\left|\boldsymbol{B} v_{k}\right|_{\psi} \leqslant k^{-1}\left|\boldsymbol{A} v_{k}\right|_{w} \rightarrow 0$ so $\boldsymbol{B} v_{k} \rightarrow 0$. Then $\boldsymbol{B} v_{*}=0$ whence also $\boldsymbol{A} v_{*}=0$, contradicting $0<\varepsilon_{*}=\varepsilon_{*}\left|v_{k}\right| \gamma \leqslant\left|\boldsymbol{A} v_{k}\right| q_{i} \rightarrow\left|\boldsymbol{A} v_{*}\right| q_{u}$.

$\S$ This is for $d>2$. For $d=1$ we could take $\mathscr{P}=\mathscr{Y}$ as in [4] while for $d=2$ we take any $\bar{q}>1$ and continue.

$\|$ This is valid pointwise for $a, R, U$ smooth. 
where $\boldsymbol{M}_{0}$ is the ordinary differential operator given formally on $(0,1)$ by

$$
M_{0}: f \mapsto-\left(a f^{\prime}\right)^{\prime}-\frac{d-1}{r} a f^{\prime}
$$

and $S$ is a second-order elliptic operator, acting as a densely defined, self-adjoint, semi-definite operator on $u:=L^{2}\left(S^{d-1}\right)$ with compact resolvent.

A significant observation is that the spherical operator $S$ does not depend on $A$, i.e. on the particular choice of $a(\cdot), \gamma$ in (1.1) and (1.2) (iii). We write $\left\{\mu_{j}: j=0,1, \ldots\right\}$ for the distinct eigenvalues of $S$ so

$$
0=\mu_{0}<\mu_{1}<\ldots \rightarrow \infty
$$

and, for each $\mu=\mu_{j}$, we let

$$
U_{\mu}:=\{U: S U=\mu U\} \subset \mathcal{U}:=L^{2}\left(S^{d-1}\right)
$$

be the corresponding eigenspace. Note that each $u_{\mu}$ is finite dimensional and that the elements of $U_{\mu}$ (eigenfunctions of $S$ ) are just the classical 'angular' functions. The subspaces $\left\{u_{\mu}\right\}$ are orthogonal, giving a direct sum decomposition:

$$
u=\left\{u_{\mu}: \mu=0, \mu_{1}, \ldots\right\} \text {. }
$$

Now let $\mathscr{X}$ be the weighted $L^{2}(0,1)$ with the inner product

$$
\langle x, y\rangle_{\mathscr{X}}:=\int_{0}^{1} r^{d-1} x(r) y(r) \mathrm{d} r
$$

and corresponding norm. For $\mu=0, \mu_{1}, \ldots$ we set

$$
\begin{aligned}
\mathscr{H}_{\mu} & :=\mathscr{X} \otimes \mathcal{U}_{\mu} \quad \text { (tensor product) } \\
& :=\overline{\operatorname{sp}}\left\{u=R(r) U(w): R \in \mathscr{X}, U \in \mathcal{U}_{\mu}\right\} \\
& =\left\{\sum_{J=1}^{J(\mu)} R_{j}(r) \bar{U}_{j}(\omega): R_{j} \in \mathscr{X}\right\} \subset \mathscr{H}:=L^{2}(\Omega)
\end{aligned}
$$

where $\left\{\bar{U}_{j}=\bar{U}_{\mu, j} ; j=1, \ldots, J(\mu)\right\}$ is an orthonormal basis for $\boldsymbol{U}_{\mu}$. Corresponding to (2.19) we then have an orthogonal direct sum decomposition:

$$
\mathscr{H}=\oplus\left\{\mathscr{H}_{\mu}: \mu=0, \mu_{1}, \ldots\right\} .
$$

Note that the norm $|\cdot|_{\mathscr{Q}}$ corresponding to (2.20) gives $|R U|_{\mathscr{H}}=|R|_{\mathscr{L}}|U|_{\mathscr{Q}}$ for (2.15) and if, corresponding to (2.21) and (2.22), we consider $u, v \in \mathscr{H}$ expanded as

$$
u=\sum_{\mu} \sum_{j=1}^{J(\mu)} R_{\mu, j} \bar{U}_{\mu, j} \quad v=\sum_{\mu} \sum_{j=1}^{J(\mu)} \hat{R}_{\mu, j} \bar{U}_{\mu, j}
$$

with $R_{\mu, j}, \hat{R}_{\mu, j} \in \mathscr{X}$, then

$$
\langle u, v\rangle_{\mathscr{X}}=\sum_{\mu} \sum_{j=1}^{J(\mu)}\left\langle R_{\mu, j}, \hat{R}_{\mu, j}\right\rangle_{\mathscr{X}}
$$


in view of the orthonormality of $\left\{\tilde{U}_{\mu, j}: j=1, \ldots, J(\mu): \mu=0, \mu_{1}, \ldots\right\}$. Note that for $u$ as in (2.15) with $U \in u_{\mu}$ (e.g. $U=\bar{U}_{\mu, j}$ ) we have

$$
\boldsymbol{L}_{\psi} u=\left[\left(\boldsymbol{M}_{0}+\mu a / r^{2}+\psi\right) R\right] U
$$

and we are led to analyse $\boldsymbol{M}_{\mu}:=\boldsymbol{M}_{0}+\mu a / r^{2}\left(\mu=\mu_{0}, \mu_{1}, \ldots\right)$.

Integrating by parts and using the boundary conditions (1.2) (iii), we obtain the weak formulation of $\boldsymbol{M}_{\mu}$ :

$$
\left\langle\boldsymbol{M}_{\mu} f, g\right\rangle_{\mathscr{X}}=\int_{0}^{1} r^{d-1} a(r)\left(f^{\prime} g^{\prime}+\frac{\mu}{r^{2}} f g\right) \mathrm{d} r-\gamma f(1) g(1) .
$$

To proceed it is necessary to distinguish the two cases: $\mu=\mu_{0}=0$ and $\mu=$ $\mu_{1}, \mu_{2}, \ldots>0$. In each case we take $9 y$ to be a weighted $H^{1}$ space, but use slightly different norms. We take $\mathscr{Y}_{0}, \mathscr{y}_{+}$to be the Hilbert spaces of functions $f$ on $(0,1)$ induced $\dagger$ respectively, by the norms

$$
\begin{aligned}
& \|f\|_{0}:=\left(\int_{0}^{1} r^{d-1}\left(\left|f^{\prime}\right|^{2}+|f|^{2}\right) \mathrm{d} r\right)^{1 / 2} \\
& \|f\|_{+}:=\left(\int_{0}^{1} r^{d-1}\left(\left|f^{\prime}\right|^{2}+\mu_{1} r^{d-3}|f|^{2}\right) \mathrm{d} r\right)^{1 / 2} .
\end{aligned}
$$

Observe that $\|\cdot\|_{+}$dominates $\|\cdot\|_{0}$ since $r^{-2}>1$ and $\mu_{1}>0$ so $y_{+} \subset \mathscr{Y}_{0}$ with, clearly, a dense embedding. We complete the weak formulation of

$$
M_{\mu}: y \rightarrow y^{*} \quad\left(\mathscr{Y}:=y_{0} \text { for } \mu=0 ; y=y_{+} \text {for } \mu>0\right)
$$

by specifying that $(2.23)$ is to hold for $f, g \in \mathcal{Y}$, as appropriate.

From standard embedding results [5], one easily sees that $\mathscr{Y}_{0}$ (a fortiori $\mathscr{Y}_{+}$) embeds in $C^{1 / 2}[\bar{r}, 1]$ for any $\bar{r}>0$ so one has an estimate

$$
|f(1)| \leqslant C_{1}\|f\|_{0} \quad \text { for } f \in \mathscr{Y}_{0} \text {. }
$$

Also, $y$ embeds compactly in $C[\bar{r}, 1]$ for $\bar{r}>0$ from which it follows, as earlier for (2.12), that

$$
|f(1)|^{2} \leqslant \varepsilon\|f\|_{0}^{2}+C_{\varepsilon}|f|_{\dddot{2}}^{2} \quad \text { for } f \in \mathscr{Y}_{0}
$$

for any $\varepsilon>0$. We will need more precise information about the behaviour of $f \in \mathscr{Y}_{0}$ as $r \rightarrow 0+$. For $0<r<1$ we have

$$
\begin{aligned}
|f(1)-f(r)| & =\left|\int_{r}^{1} s^{-(d-1) / 2}\left[s^{(d-1) / 2} f^{\prime}(s)\right] \mathrm{d} s\right| \\
& \leqslant\left(\int_{r}^{1} s^{1-d} \mathrm{~d} s\right)^{1 / 2}\|f\|_{0}
\end{aligned}
$$

† In defining these spaces there are three considerations at issue: regularity in $(0,1)$, behaviour near 0 and behaviour at the boundary. The norms in (2.24) take care of the first two of these in taking the closure of the set of smooth functions. For first-order boundary conditions one can consider smooth functions satisfying (1.2) (iii) pointwise when $a(\cdot)$ is smooth near 1 and get 'all' of $H(0,1]$ near the boundary; this is independent of (smooth) $a(\cdot)$ and is also correct for $a(\cdot)$ merely measurable/bounded as in (1.3). (If we were to consider Dirichlet conditions then the specification of $y$ would include the requirement that $f(1)=0$.) For $\mu=0$ we thus have $\varkappa_{0}=$ constants on the unit sphere $\left.S^{d-1}\right\}$ so $\mathscr{H}_{0}$ is just the space of all radial functions in $L^{2}(\Omega)$ while (2.24) makes $Y_{0} \otimes U_{0}$ the subspace of radial functions in $\mathscr{V}=H^{1}(\Omega)$ (in $\mathscr{V}=H_{0}^{1}(\Omega)$ in the case of Dirichlet conditions) with a norm isometry. And so on. 
whence, as $|f(r)| \leqslant|f(1)|+|f(1)-f(r)|$, we have

$$
|f(r)| \leqslant \begin{cases}\left(C_{1}+\ln ^{1 / 2} r\right)\|f\|_{0} & d=2 \\ C_{*} r^{1-d / 2}\|f\|_{0} & d=3,4, \ldots\end{cases}
$$

with $C_{1}, C_{*}$ depending only on $d$. If we define $\mathscr{E}_{v}$ as the space of functions continuous on $(0,1]$ for which the norm

$$
|f|_{[v]}:=\sup \left\{r^{\nu / 2}|f(r)|: 0<r \leqslant 1\right\}
$$

is finite, then (2.26) shows that $\mathscr{Y}_{0}$ embeds (continuously, by the closed graph theorem) in $\mathscr{Z}_{v}$ for $v \geqslant \bar{v}:=d-2(v>\bar{v}=0$ for $d=2)$.

Lemma 3. $\mathscr{Y}_{0}$ embeds compactly in $\mathscr{W}_{v}$ for $v>\bar{v}:=d-2(d \geqslant 2)$.

Proof. Suppose $d>2$ and $\left\{f_{k}\right\}$ is bounded in $\mathscr{Y}_{0}$. We can extract a subsequence (again denoted by $\left\{f_{k}\right\}$ ) converging $\mathscr{Y}_{0}$-weakly, say to $\bar{f}$, and we will show $f_{k} \rightarrow \bar{f}$ in $\mathscr{Z}_{v}$. Note that $f_{k} \rightarrow \bar{f}$ uniformly on $[\bar{r}, 1]$ for each $\bar{r}>0$ since the embedding: $\mathscr{Y}_{0} \rightarrow C[\bar{r}, 1]$ is compact (as $\|\cdot\|_{0}$ dominates the $H^{1}(\bar{r}, 1)$ norm); cf, e.g., [5]. Since $v>\bar{v}$ we have, by (2.26)

$$
r^{v^{\prime 2}}\left|f_{k}(r)-\bar{f}(r)\right| \leqslant C_{*} r^{v^{\prime}}\left\|f_{k}-\bar{f}\right\|_{0} \leqslant \bar{C} r^{v^{\prime}}
$$

with $2 v^{\prime}=v-\bar{v}>0$ and with $\tilde{C}$ fixed for the sequence. Given any $\varepsilon>0$ we can choose $\bar{r}$ so the right-hand side of $(2.28)$ is less than $\varepsilon$ on $(0, \bar{r})$. Then, noting the uniform convergence $f_{k} \rightarrow \bar{f}$ on $[\tilde{r}, 1]$, we can choose $K=K(\varepsilon)$ large enough that the left-hand side of (2.28) is less than $\varepsilon$ on $[\bar{r}, 1]$ for each $k \geqslant K$, giving $\left|f_{k}-f\right|_{[\nu]} \leqslant \varepsilon$.

For $d=2$ we may take any $\tilde{v} \in(0, v)$ and note that $(2.26)$ gives

$$
|f(r)| \leqslant C_{*} r^{-\bar{\nu} / 2}\|f\|_{0}
$$

( $C_{*}$ now depending on the choice of $\tilde{v}$ ), giving (2.28) with $2 v^{\prime}=v-\tilde{v}$. The proof concludes as before.

Next, we consider the weighted $H^{1}(0,1)$ spaces $\hat{y}_{v}$ induced by the norms

$$
\|f\|_{[\nu]}:=\left(\int_{0}^{1} r^{r}\left(\left|f^{\prime}\right|^{2}+|f|^{2}\right) \mathrm{d} r\right)^{1 / 2}
$$

for $v \geqslant 0$; observe that $\mathscr{Y}_{0}=\mathscr{Y}_{d-1}$. We will set $\mathscr{P}:=\hat{\mathscr{Y}}_{\dot{v}}$ with $\bar{v}:=2 d-3$ for $d>2$ (any $\bar{v}>1$ for $d=2$ ).

Lemma 4. Let $\varphi \in\left(\hat{\mathscr{Y}}_{p}\right) *$ for some $v>2 d-3(d \geqslant 2)$. Then (2.7) holds with $\mathscr{y}=\mathscr{Y}_{0}(a$ fortiori with $\mathscr{Y}=\mathscr{Y}_{+}$).

Proof. Set $\tilde{v}=v-(d-1)$ so $v>2 d-3$, as assumed, gives $\tilde{v}>d-2$ whence, by lemma 3, the embedding: $\mathscr{Y}_{0} \hookrightarrow \mathscr{Z}_{i}$ is compact. Again this gives an estimate

$$
r^{v}|f(r)|^{2} \leqslant \hat{\varepsilon}\|f\|_{0}^{2}+\hat{C}|f|_{\mathscr{P}}^{2} \quad \text { for } f \in \mathscr{Y}_{0} \subset \mathscr{X}
$$


for arbitrary $\hat{\varepsilon}>0$ and with $\hat{C}$ depending on $\hat{\varepsilon}, v, d$. We have $\left(f^{2}\right)^{\prime}=2 f f^{\prime}$ so

$$
\begin{aligned}
& \int r^{\nu}\left|\left(f^{2}\right)^{\prime}\right|^{2} \mathrm{~d} r \leqslant 4 \sup \left\{r^{\tilde{y}}|f(r)|^{2}\right\} \int r^{d-1}\left|f^{\prime}(r)\right|^{2} \mathrm{~d} r \\
& \leqslant 4\left(\hat{\varepsilon}|| f \|_{0}^{2}+\hat{C}|f|_{\mathscr{x}}^{2}\right)\left|f^{\prime}\right|_{\mathscr{x}}^{2} \\
& \int r^{\nu}\left|f^{2}(r)\right|^{2} \mathrm{~d} r \leqslant\left(\hat{\varepsilon}\|f\|_{0}^{2}+\hat{C}|f|_{\mathscr{x}}^{2}\right)|f|_{\mathscr{2}}^{2} \\
& \left\|f^{2}\right\|_{[v]}^{2} \leqslant 4\left(\hat{\varepsilon} \|\left. f\right|_{0} ^{2}+\hat{C}|f|_{\mathscr{x}}^{2}\right)\|f\|_{0}^{2} .
\end{aligned}
$$

Setting $4 \hat{\varepsilon}:=\varepsilon^{2}$ (also fixing $\hat{C}$ ) we then have

$$
\left\|f^{2}\right\|_{[y]} \leqslant \varepsilon\|f\|_{0}^{2}+C|f|_{\mathscr{x}}^{2}
$$

for any $C$ large enough that $2 \varepsilon C \geqslant \hat{\varepsilon}+4 \hat{C}$ and also $C^{2} \geqslant \hat{C}$.

For $\varphi \in\left(\hat{\mathrm{Y}}_{\nu}\right)^{*}$ let $M:=\left[\left(\hat{\mathrm{Y}}_{\nu}\right)^{*}-\right.$ norm of $\left.\varphi\right]<\infty$ and note that (2.31) gives

$$
|\langle\varphi x, x\rangle|=\left|\left\langle\varphi, x^{2}\right\rangle\right| \leqslant M \|\left. x^{2}\right|_{[v]} \leqslant(\varepsilon M)|x|_{\mathscr{y}}^{2}+(C M)|x|_{\mathscr{Z}}^{2}
$$

for $x \in \mathscr{Y}=\mathscr{Y}_{0}\left(\right.$ or $\left.\mathscr{Y}_{+} \subset \mathscr{Y}_{0}\right)$ and any $\varepsilon M>0$ and correspondingly determined $C M$. This is just (2.7).

Lemma 5. With $\mathscr{P}:=\hat{\mathscr{Y}}_{\vec{v}}$, as above, we have (2.5) with $\mathscr{Y}=\mathscr{Y}_{0}\left(\right.$ a fortiori with $\left.\mathscr{Y}=\mathscr{Y}_{+}\right)$.

Proof. Note that $\tilde{v}:=\bar{v}-(d-1)>0$ for any $d \geqslant 2$. We no longer have compactness but (2.26) gives

$$
r^{2}|f(r)|^{2} \leqslant C_{*}|f|_{9}^{2} \quad \text { for } f \in \mathscr{Y}
$$

corresponding to (2.30) so we obtain, as for (2.31), the estimate

$$
\left|x^{2}\right|_{\mathscr{p} p}:=\left\|x^{2}\right\|_{[\tilde{y}]} \leqslant C^{*}|x|_{\mathscr{y}}^{2} \quad \text { for } x \in \mathcal{O} y
$$

absorbing $|x|_{\mathscr{x}}$ terms in $|x|_{y}$. From the identity

$$
x y=\left[(c x+y / c)^{2}-(c x-y / c)^{2}\right] / 4
$$

we then obtain

$$
\begin{aligned}
|x y| & \leqslant\left(C^{*} / 4\right)\left(|c x+y / c|_{y}^{2}+|c x-y / c|_{y}^{2}\right) \\
& \leqslant\left(C^{*} / 2\right)\left(c|x|_{y}+|y|_{y} / c\right)^{2}
\end{aligned}
$$

which is just (2.5) with $\bar{C}:=2 C^{*}$ on setting $c^{2}:=|y|_{9 y} /\left.x\right|_{y}$.

With these lemmas in hand we are ready to proceed to the construction (2.2) (iii).

Theorem 6. Let $a(\cdot)$ satisfy (1.3) and consider the boundary conditions (1.2) (iii). With $\mathscr{P}:=\hat{Y}_{\bar{v}}$ as above, assume $\psi \in \mathscr{P}^{*}$ with $\psi \geqslant \varphi$ for some $\varphi$ as in lemma 4 . Then $\boldsymbol{L}_{\psi}=\boldsymbol{A}+\boldsymbol{\psi}$ induces a closed, densely defined, self-adjoint operator $\boldsymbol{A}_{\psi}$ on $\mathscr{H}$, as in (2.1).

Proof. We begin by considering the ordinary differential operator $\left(\boldsymbol{M}_{\mu}+\boldsymbol{\psi}\right)$, first for $\mu>0$ so we are taking $y=y_{+}$and $|\cdot|_{y}=\mid \cdot \|_{+}$. From (2.23), using (1.3) and (2.25), we 
have

$$
\begin{aligned}
\left\langle\boldsymbol{M}_{\mu} x, x\right\rangle & \geqslant \alpha \int_{0}^{1}\left(r^{d-1}\left|x^{\prime}\right|^{2}+\mu r^{d-3}|x|^{2}\right) \mathrm{d} r-\gamma_{+}|x(1)|^{2} \\
& \geqslant \alpha|x|_{\mathscr{Y}}^{2}+\left(\mu-\mu_{1}\right) \alpha|x|_{\mathscr{Q}}^{2}-\gamma_{+}\left(\varepsilon|x|_{\mathscr{O}}^{2}+C_{\varepsilon}|x|_{\mathscr{Q}}^{2}\right)
\end{aligned}
$$

where $\gamma_{+}:=\max \{\gamma, 0\}$. Choose $\varepsilon<\alpha / \gamma_{+}$(thus also fixing $C_{\varepsilon}$ ) and one obtains (for $\mu \geqslant 1$ ) the monotonicity estimate $\uparrow$

$$
\left\langle\boldsymbol{M}_{\mu} x, x\right\rangle \geqslant \underline{\alpha}|x|_{\mathscr{Y}}^{2}+\left(\alpha \mu-\beta_{0}\right)|x|_{\mathscr{L}}^{2} \quad \text { for } x \in \mathcal{Y}=\mathscr{Y}_{+}
$$

where $\alpha:=\alpha-\gamma_{+}^{\epsilon}$ and $\beta_{0}:=\alpha \mu_{1}+\gamma_{+} C_{\varepsilon}$, i.e. (2.3) holds. The symmetry condition (2.4) is clear from (2.23) and lemmas 3 and 4 ensure the hypotheses on $\psi, \varphi$ for applicability of lemma 2. Thus we know that for each $\mu=\mu_{1}, \mu_{2}, \ldots$ there is a well defined self-adjoint operator

$$
\boldsymbol{M}_{\mu, \psi}: \mathscr{X} \supset \mathscr{D}_{\mu, \psi} \rightarrow \mathscr{X}
$$

which maps: $x \mapsto(M x+\psi x) \in \mathscr{X}$ whenever $x \in \mathscr{D}_{\mu, \psi} \subset \mathscr{Y}_{+} \subset \mathscr{X}$. For $\mu=\mu_{0}=0$, taking $\circlearrowleft=\vartheta_{0}$ and $|\cdot|_{y}=\|\cdot\|_{0}$, one similarly obtains $(2.3)$ with $\underline{\alpha}$ and $\beta=\beta_{0}$ exactly as in (2.32). Thus, we have (2.33) for every $\mu=\mu_{0}, \mu_{1}, \ldots$

At this point we recall $(2.21),(2.22)$ and note that each $u \in \mathscr{H}$ has the orthogonal expansion

$$
u(x)=\sum_{\mu} \sum_{j=1}^{J(\mu)} R_{\mu, j}(r) \bar{U}_{\mu, j}(\omega) \quad(x=r \omega)
$$

where the outer sum is over $\mu=\mu_{0}, \mu_{1}, \ldots$ and each $R_{\mu, j}$ is in $\mathscr{X}$. The orthonormality of $\left\{\tilde{U}_{\mu, j}\right\}$ gives the norm identity

$$
|u|_{\mathscr{H}}^{2}=\sum_{\mu} \sum_{j=1}^{J(\mu)}\left|R_{\mu, j}\right|_{\mathscr{\mathscr { E }}}^{2}
$$

with a corresponding formula for the $\mathscr{H}$ inner product. We now define, in terms of (2.33),

$$
\boldsymbol{A}_{\psi} u:=\sum_{\mu} \sum_{j=1}^{J(\mu)}\left[M_{\mu, \psi} R_{\mu, j}\right] \bar{U}_{\mu, j}
$$

for $u$, given by $(2.34)$, in

$$
\mathscr{D}_{\psi}:=\left\{u \in \mathscr{H}: \text { each } R_{\mu, j} \text { of (2.34) is in } \mathscr{D}_{\mu, \psi} \text { with } \sum_{\mu} \sum_{j=1}^{J(\mu)}\left|M_{\mu, \psi} R_{\mu, j}\right|_{\mathscr{X}}^{2}<\infty\right\} .
$$

It is easy to verify that this definition of $\boldsymbol{A}_{\psi}, \mathscr{D}_{\psi}$ is independent of the particular choices of orthonormal bases $\left\{\bar{U}_{\mu, j}: j=1, \ldots, J(\mu)\right\}$ made for each $u_{\mu}$.

Since each $\mathscr{D}_{\mu . \psi}$ is dense in $\mathscr{X}$ we have the set finite sums (2.34) with each $\left.R_{\mu, j} \in \mathscr{D}_{\mu, \psi}\right\}$ dense in $\mathscr{H}$ so $\boldsymbol{A}_{\psi}$ is densely defined. If $u^{k} \rightarrow \tilde{u}$ in $\mathscr{H}$ (with each $u^{k} \in \mathscr{D}_{\psi}$ ) and $\nmid$ We only need (2.3) immediately but emphasise that $\underline{\alpha}$ and $\beta_{0}$ are independent of $\mu$ for $\mu=\mu_{1}, \mu_{2}, \ldots$ 
also $A_{\psi} u^{k} \rightarrow w$ in $\mathscr{H}$, then each $R_{\mu}^{k}, j \rightarrow \tilde{R}_{\mu, j}$ in $\mathscr{X}$ and $\boldsymbol{M}_{\mu, \psi} R_{\mu, j}^{k}$ converges in $\mathscr{X}$, necessarily to $\boldsymbol{M}_{\mu, \psi} \tilde{R}_{\mu, j}$, with

$$
\sum_{\mu} \sum_{j}\left|\boldsymbol{M}_{\mu, j} \tilde{R}_{\mu, j}\right|^{2}=|w|_{\mathscr{H}}^{2}<\infty
$$

so $\tilde{u} \in \mathscr{D}_{\psi}$ with $\boldsymbol{A}_{\psi} \tilde{u}=w:=\lim _{k} \boldsymbol{A}_{\psi} u^{k}$, i.e. $\boldsymbol{A}_{\psi}$ is closed. Next, suppose $\tilde{u} \in \mathscr{D}\left(\boldsymbol{A}_{\psi}^{*}\right)$, meaning $\mathscr{H}$-continuity (on $\mathscr{D}_{\psi}$ ) of the functional: $u \rightarrow\left\langle\boldsymbol{A}_{\psi} u, \tilde{u}\right\rangle$ so we have an identity $\left\langle A_{\psi} u, \tilde{u}\right\rangle=\langle u, \hat{u}\rangle$ for some $\hat{u} \in \mathcal{H}$. With the obvious notation, taking $u:=R_{\mu, j} \bar{U}_{\mu, j}$ gives

$$
\left\langle M_{\mu, \psi} R_{\mu, j}, \tilde{R}_{\mu, j}\right\rangle_{\mathscr{X}}=\left\langle R_{\mu, j}, \hat{R}_{\mu, j}\right\rangle \quad \text { for } R_{\mu, j} \in \mathscr{D}_{\mu, \psi}
$$

whence, as $\boldsymbol{M}_{\mu, \psi}$ is self-adjoint, we have $\tilde{R}_{\mu, j} \in \mathscr{D}_{\mu, \psi}$ and $\hat{R}_{\mu, j}=\boldsymbol{M}_{\mu, \psi} \tilde{R}_{\mu, j}$. As in (2.35), this (for each $\mu, j$ ) gives

$$
\sum_{\mu} \sum_{j}\left|\boldsymbol{M}_{\mu, j} \tilde{R}_{\mu, j}\right|^{2}=|\hat{u}|_{\mathscr{H}}<\infty
$$

whence $\hat{u} \in \mathscr{D}_{\psi}$. Thus $\mathscr{D}\left(\boldsymbol{A}_{\psi}^{*}\right) \subset \mathscr{D}_{\psi}=: \mathscr{D}\left(\boldsymbol{A}_{\psi}\right)$. Since one obviously has the reverse inclusion, it follows that $\boldsymbol{A}_{\psi}$ is self-adjoint.

We remark that $\boldsymbol{A}_{\psi}$, as defined in (2.36), has compact resolvent but it is convenient to defer proof of this until the discussion of spectral analysis of $\boldsymbol{A}_{\psi}$ in the next section. The final task of this section is verification of the consistency of (2.2) (ii) and (iii).

Lemma 7. Suppose $\boldsymbol{A}_{\psi}, \mathscr{D}_{\psi}$ are defined as in (2.14), directly by application of theorem 2 , and also as in (2.36). Then these definitions are equivalent.

Proof. For $u$ of the form (2.15) with $U \in \mathcal{U}_{\mu}$ we have $u \in \mathscr{D}_{\psi}^{(\mathrm{ii})}$ if and only if $R \in \mathscr{D}_{\mu, \psi} \subset$ $\mathscr{Y}_{0}$. Since elements of $\mathcal{u}_{\mu}$ are smooth, this gives $u \in \mathcal{V}$ and, from (2.16), etc, we have

$$
(\boldsymbol{A}+\psi+\lambda)[R U]=\left[\left(\boldsymbol{M}_{\mu}+\psi+\lambda\right) R\right] U=: \hat{R} U+\lambda u
$$

so $\boldsymbol{M}_{\mu, \psi} R:=\hat{R} \in \mathscr{X}$ implies $R U \in \mathscr{D}_{\psi}^{(\mathrm{ii})}$ and $\boldsymbol{A}_{\psi}^{(\mathrm{ii})} u=\hat{R} U=\boldsymbol{A}_{\psi}^{(\mathrm{iii})} u$. Conversely, $R U \in \mathscr{D}_{\psi}^{(\mathrm{ii})}$ means $(\hat{R}+\lambda) U \in \mathscr{H}$ whence $\hat{R} \in \mathscr{X}$ so $R \in \mathscr{D}_{\mu, \psi}$. For either of the definitions one obtains a closed operator and the span of such $u=R U$ is dense in each graph. Thus, the definitions of $\boldsymbol{A}_{\psi}, \mathscr{D}_{\psi}$ coincide.

\section{Spectral theory: continuity}

We will be considering the eigenvalues of $\boldsymbol{A}_{\psi}$ (defined as in theorem 6 for $\psi \in \mathscr{P}^{*}$ with suitable lower bound $\varphi$ ), taken in increasing order with multiplicities

$$
\lambda_{1}(\psi) \leqslant \lambda_{2}(\psi) \leqslant \ldots \rightarrow \infty
$$

as a sequence of nonlinear functionals of $\psi$. The principal result of this section, after verifying (3.1), is that each functional: $\psi \mapsto \lambda_{k}(\psi)$ is continuous, topologising $\psi$ in $P^{*}$ (with a suitable one-sided estimate: $\psi \geqslant \varphi$ ).

From the proof of theorem 6 (and under those hypotheses) we know that each of the operators $\boldsymbol{M}_{\mu, \psi}\left(\mu=0, \mu_{1}, \ldots\right)$ is self-adjoint with compact resolvent and so we 
have eigenpairs $\dagger\left\{\left[\sigma_{\mu, k}, y_{\mu, k}\right]: k=1,2, \ldots\right\}$ such that, for each $\mu=0, \mu_{1}, \ldots$,

$$
\begin{aligned}
& \left\{y_{\mu, k}: k=1, \ldots\right\} \text { is an orthonormal basis for } \mathscr{X} ; \\
& \sigma_{\mu, 1} \leqslant \sigma_{\mu, 2} \leqslant \ldots \rightarrow \infty
\end{aligned}
$$

Lemma 8. Each of the functions $y_{\mu, k}(r) \bar{U}_{\mu, j}$ (for $\mu=0, \mu_{1}, \ldots ; k=1,2, \ldots ; j=1, \ldots$, $J(\mu)$ ) is an eigenfunction of $\boldsymbol{A}_{\psi}$ with corresponding eigenvalue $\sigma_{\mu, k}$. This set of functions is an orthonormal basis for $\mathscr{H}$.

Proof. For $y, \hat{y} \in \mathscr{X}$ and $U, \hat{U} \in \mathcal{U}$ we have

$$
\langle y U, \hat{y} \hat{U}\rangle_{\mathscr{H}}=\langle y, \hat{y}\rangle_{\mathscr{X}}\langle U, \hat{U}\rangle_{\varkappa}
$$

so, since $\left\{\bar{U}_{\mu, j}: \mu=0, \mu_{1}, \ldots ; j=1, \ldots, J(\mu)\right\}$ is an orthonormal basis for $u$ and each $\left\{y_{\mu, k}: k=1, \ldots\right\}$ is an orthonormal basis for $\mathscr{X}$, it follows that $\left\{y_{\mu, k} \bar{U}_{\mu, j}\right\}$ is an orthonormal basis for $\mathscr{H}=\mathscr{Q} \otimes U$. Our construction of $\boldsymbol{A}_{\psi}$ gives

$$
\begin{aligned}
& A_{\psi}(y U):=\left(M_{\mu, \psi} y\right) U \quad \text { for } U \in \mathcal{U}_{\mu}, y \in \mathscr{D}_{\mu, \psi} \\
& \boldsymbol{A}_{\psi}\left(y_{\mu, k} \bar{U}_{\mu, j}\right)=\left(M_{\mu, \psi} y_{\mu, k}\right) \bar{U}_{\mu, j}=\left(\sigma_{\mu, k} y_{\mu, k}\right) \bar{U}_{\mu, j}
\end{aligned}
$$

so each $\left(y_{\mu, k} \bar{U}_{\mu, j}\right)$ is an eigenfunction of $\boldsymbol{A}_{\psi}$.

Lemma 9. For any $\hat{\lambda} \in \mathbb{R}$ there are only finitely many $\mu \in \sigma(\boldsymbol{S})$ for which $\sigma\left(\boldsymbol{M}_{\mu, \psi}\right) \cap$ $(-\infty, \hat{\lambda}]$ is non-empty so (counting multiplicities in $\left.\sigma\left(\boldsymbol{A}_{\psi}\right)\right)$ the set $\left\{\sigma_{\mu, k}: \sigma_{\mu, k} \leqslant \lambda\right\}$ is finite.

Proof. Suppose $\sigma \leqslant \hat{\lambda}$ is an eigenvalue of $\boldsymbol{M}_{\mu, \psi}$ with corresponding eigenfunction $x \in \mathscr{D}_{\mu, \psi} \subset \mathscr{X}$, normalised so $|x|_{\mathscr{X}}=1$. Then

$$
\begin{aligned}
\sigma & =\left\langle\boldsymbol{M}_{\mu, \psi} x, x\right\rangle \\
& =\left\langle\boldsymbol{M}_{\mu} x, x\right\rangle+\langle\varphi x, x\rangle+\left\langle\psi-\varphi, x^{2}\right\rangle \\
& \geqslant\left\langle\boldsymbol{M}_{\mu} x, x\right\rangle-\|\varphi\|\left\|x^{2}\right\|_{[v]}
\end{aligned}
$$

where we take $\varphi$ in $\left(\hat{y}_{v}\right)^{*}$. From (2.32) and (2.31)

$$
\sigma \geqslant \underline{\alpha}|x|_{y}^{2}+\left(\alpha \mu-\beta_{0}\right)-\|\varphi\|\left(\varepsilon|x|_{o y}^{2}+C_{\varepsilon}\right) \text {. }
$$

Choosing $\varepsilon:=\underline{\alpha} /\|\varphi\|$, this gives

$$
\mu \leqslant\left(\sigma+\beta_{0}+\|\varphi\| C_{\varepsilon}\right) / \alpha \text {. }
$$

Since $\alpha, \underline{\alpha}, \beta_{0},\|\varphi\|, C_{\varepsilon}$ are independent of $\sigma, \mu$, we see that a bound $\hat{\lambda}$ on $\sigma$ bounds $\mu$. Since we only consider $\mu \in \sigma(\boldsymbol{L})=\left\{0, \mu_{1}, \ldots\right\}$, this restricts us to a finite set.

In particular, given a number $\hat{\sigma}$, the set $\mathcal{M}(\hat{\sigma}):=\left\{\mu \in \sigma(S): \hat{\sigma} \in \sigma\left(\boldsymbol{M}_{\mu, \psi}\right)\right\}$ is finite. Further, $\hat{\sigma}$ occurs with finite multiplicity $K_{\mu}$ for each $\mu \in \mathcal{M}(\hat{\sigma})$. For each occurrence of

$\dagger$ That is, $y_{\mu, k} \in \mathscr{D}_{\mu, \psi} \subset \mathscr{X}$ with $\boldsymbol{M}_{\mu, \psi} y_{\mu, k}=\sigma_{\mu, k} y_{\mu, k}$. (We are using $\sigma_{\text {n }}$ to denote the eigenvalues of $\boldsymbol{M}_{\mu, \psi}$ to avoid confusion with the eigenvalues $\left\{\mu_{0}, \ldots\right\}$ of $S$ or $\left\{\lambda_{1}, \ldots\right\}$ of $\boldsymbol{A}_{\psi}$. We remark at this point that in standard Sturm-Liouville theory one shows, using properties of the initial value problem for $\left(\boldsymbol{M}_{0}+\mu a / r^{2}+\psi\right) y=0$, that these eigenvalues are simple (strict inequalities in (3.2) (ii)) with certain nodal properties for the eigenfunctions. For $\psi$ as rough as here it is not clear that this remains valid.

$\$$ Note that $C_{\varepsilon}$ here, coming from (2.31) in lemma 5, depends only on $\underline{\alpha}$ and $\|\varphi\|$ whereas the $C_{\varepsilon}$ appearing in the definition of $\beta_{0}$ for (2.32) comes from (2.25), depending only on the relation of $\underline{\alpha}$ to the $\alpha$ in (1.3). 
$\hat{\sigma}$ in $\left[\sigma\left(\boldsymbol{M}_{\mu, \psi}\right)\right.$ with multiplicities], say with eigenfunction $\hat{y}$, it occurs $J(\mu)$ times in $\left[\sigma\left(\boldsymbol{A}_{\psi}\right)\right.$ with multiplicities $]$ - with corresponding eigenfunctions $\left\{\hat{y} \tilde{U}_{\mu, j}: j=1, \ldots, J(\mu)\right\}$. Thus, the set $\left\{y_{\mu, k} \vec{U}_{\mu, j}\right\}$ contains exactly

$$
m_{\hat{\sigma}}:=\sum_{\mu \in \mathscr{M}(\hat{\sigma})} J(\mu) K_{\mu}
$$

eigenfunctions associated with $\hat{\sigma}$ as an eigenvalue of $\boldsymbol{A}_{\psi}$.

From the above it follows that $\left\{\sigma_{\mu, k}\right\}$ is a discrete set which, when sorted in increasing order (with multiplicities), we can relabel as $\left(\lambda_{1}, \lambda_{2}, \ldots\right)$ with $\lambda_{k} \rightarrow \infty$ as in (3.1) with an associated orthonormal basis for $\mathscr{H}$ consisting of eigenfunctions $\dagger$ of $\boldsymbol{A}_{\psi}$ which we relabel as $\left\{w_{k}=w_{k}(\psi): k=1,2, \ldots\right\}$ so $\boldsymbol{A}_{\psi} w_{k}=\lambda_{k} w_{k}$. It follows that $\left[\sigma\left(\boldsymbol{A}_{\psi}\right)\right.$ with multiplicities] is precisely $\left\{\lambda_{1}, \lambda_{2}, \ldots\right\}$ with the multiplicities given by (3.4).

An immediate corollary to the spectral expansion given by the eigenpairs $\left\{\left[\lambda_{k}\right.\right.$, $\left.\left.w_{k}\right]\right\}$ :

$$
\begin{aligned}
& u=\sum_{k} v_{k} w_{k} \quad\left(|u|_{\mathscr{H}}^{2}=\sum_{k}\left|v_{k}\right|^{2}\right) \\
& \boldsymbol{A}_{\psi} u=\sum_{k}\left(\lambda_{k} v_{k}\right) w_{k} \quad \text { for } u \in \mathscr{D}_{\psi}
\end{aligned}
$$

where

$$
u \in \mathscr{D}_{\psi} \Leftrightarrow \sum_{k}\left|\lambda_{k} v_{k}\right|^{2}<\infty
$$

is that $\boldsymbol{A}_{\psi}$ has compact resolvent (since $\left(\lambda-\lambda_{k}\right)^{-1} \rightarrow 0$ for $\left.\lambda \notin \sigma\left(\boldsymbol{A}_{\psi}\right)=\left\{\lambda_{1}, \ldots\right\}\right)$. From (3.5) we easily obtain

$$
\left\langle\boldsymbol{A}_{\psi} u, u\right\rangle=\sum_{k} \lambda_{k} v_{k}^{2} \quad \text { for } u \in \mathscr{D}_{\psi} \text { as in (3.5) }
$$

and note, from this and (3.1), that $\left\langle\boldsymbol{A}_{\psi} u, u\right\rangle$ attains its minimum on

$$
\left\{u \in \mathscr{D}_{\psi}:|u|_{\mathscr{H}}=1 \text { with } u \perp w_{k} \text { for } k<K\right\}
$$

at, e.g., ${ }_{K} u=w_{K}$ with the minimum value $\lambda_{K}$. It will be convenient to obtain a slightly different recursive variational characterisation of $\lambda_{K}$. For $\mu \in \sigma(S)$ we set

$$
\mathscr{S}_{\mu, K}:=\left\{u=y \bar{U}: y \in \mathscr{Y} \text { with }|y|_{\mathscr{C}}=1 ; \bar{U} \in\left\{\tilde{U}_{\mu, j}\right\} ; u \perp w_{k} \text { for } k<K\right\}
$$

with $\mathscr{Y}:=\mathscr{Y}_{0}$ for $\mu=0$ and $\mathscr{Y}_{+}$for $\mu=\mu_{1}, \ldots$

Lemma 10. For the problem

$$
\text { minimise }\langle(\boldsymbol{A}+\psi) u, u\rangle \text { subject to: } u \in \mathrm{U}_{\mu} \mathscr{S}_{\mu, K}
$$

the minimum is attained with the minimum value $\lambda_{K}$. The minimiser $\bar{u}$ is an eigenfunction of $\boldsymbol{A}_{\psi}$ which can be taken to be $w_{K}$.

† We canonically take these to have the form $w=y \bar{U}_{\mu, j}$ (once we have fixed the orthonormal bases $\left\{\bar{U}_{\mu, j}\right\}$ for each $U_{\mu}$ ) with $y$ an eigenfunction of $\boldsymbol{M}_{\mu, \psi}$. 
Proof. Let $\hat{\lambda}:=\inf \left\{\langle(A+\psi) u, u\rangle ; u \in U_{\mu} \varphi_{\mu, K}\right\}$. We already know from (3.5) and (3.6) that $\lambda_{K}$ is attained at $u=w_{K} \in \cup_{\mu} \mathscr{S}_{\mu, K}$ so in (3.8) we need only consider $u$ for which $\langle(\boldsymbol{A}+\psi) u, u\rangle \leqslant \lambda_{K}$. As in the argument for (3.3) we then have, for $u=y \bar{U} \in \mathrm{U}_{\mu} \mathscr{S}_{\mu, K}$,

$$
\begin{gathered}
\left.\lambda_{K} \geqslant\langle\boldsymbol{A}+\psi) u, u\right\rangle=\left\langle\left(\boldsymbol{M}_{\mu}+\psi\right) y, y\right\rangle_{\mathscr{X}} \\
\quad \geqslant \underline{\alpha}|y|_{y}^{2}+\left(\alpha \mu-\beta_{0}\right)-\|\varphi\|\left(\varepsilon|y|_{y}^{2}+C_{\varepsilon}\right) \\
(\underline{\alpha} / 2 \alpha)|y|_{\mathscr{y}}^{2}+\mu \leqslant\left(\lambda_{K}+\beta_{0}+\|\varphi\| C_{\varepsilon}\right) / \alpha
\end{gathered}
$$

where we have here taken $\varepsilon=\underline{\alpha} / 2\|\varphi\|$ for use in $(2.31)$.

If we consider a minimising sequence for (3.8), we see that only finitely many $\mu \in \sigma(\boldsymbol{S})$ need be considered. As there are then only finitely many relevant $\left\{\bar{U}_{\mu, j}\right\}$, we may extract a (minimising) subsequence of the form $y_{k} \bar{U}$ with $\bar{U}=\bar{U}_{\mu, j}(\mu, j$ fixed), $y_{k} \in \mathcal{Y}$, and

$$
\left\langle(\boldsymbol{A}+\psi) u_{k}, u_{k}\right\rangle=\left\langle\left(\boldsymbol{M}_{\mu}+\psi\right) y_{k}, y_{k}\right\rangle_{\varkappa} \rightarrow \hat{\lambda} .
$$

Further, we have $\left\{\left|y_{k}\right|_{y}\right\}$ bounded so we may also assume $y_{k} \rightarrow \bar{y}$ (weak convergence in y).

Choosing $\lambda$ large enough that $\left(\boldsymbol{M}_{\mu}+\psi+\lambda\right): y \rightarrow y^{*}$ is strictly monotonic, the quadratic form $\left[y \mapsto\left\langle\left(\boldsymbol{M}_{\mu}+\psi+\lambda\right) y, y\right\rangle_{2}\right]$ is convex and so lower semicontinuous with respect to weak convergence in 9 . Thus

$$
\begin{aligned}
\left\langle\left(\boldsymbol{M}_{\mu}+\psi\right) \bar{y}, \bar{y}\right\rangle & =\left\langle\left(\boldsymbol{M}_{\mu}+\psi+\lambda\right) y, y\right\rangle-\lambda \\
& \leqslant \liminf \left\langle\left(\boldsymbol{M}_{\mu}+\psi+\lambda\right) y_{k}, y_{k}\right\rangle-\lambda \\
& =\liminf \left\langle\left(\boldsymbol{M}_{\mu}+\psi\right) y_{k}, y_{k}\right\rangle=\hat{\lambda}
\end{aligned}
$$

and the minimisation (3.8) is attained at $\bar{u}=\bar{y} \bar{U}$; set $\bar{z}:=\left(\boldsymbol{M}_{\mu}+\psi\right) \bar{y} \in \mathrm{YY}^{*}$.

Let $\left\{\bar{y}_{1}, \ldots, \hat{y}_{K}\right\}=\left\{y \in \mathscr{X}: y \bar{U} \in\left\{w_{1}, \ldots, w_{K-1}\right\}\right\}$ with $\bar{U}:=\bar{U}_{\mu, j}$ for the fixed $(\mu, j)$. Then $y \bar{U} \perp w_{k}(k<K)$ in $\mathscr{H}$ precisely when $y \perp \hat{y}_{k}\left(k=1, \ldots, K^{\prime}\right)$ in $\mathscr{X}$. We set

$$
\begin{aligned}
\mathscr{S}_{*} & =\left\{y \in \mathscr{Y}:|y|_{\mathscr{X}}=1 \text { and } y \perp y_{k}\left(k=1, \ldots, K^{\prime}\right)\right\} \\
\rho(t) & =\rho(t ; y):=\langle(\boldsymbol{M}+\psi+\lambda)(\bar{y}+t y), \bar{y}+t y\rangle_{\mathscr{X}} \quad \text { for } y \in \mathscr{Y}_{*} \\
& =(\hat{\lambda}+\lambda)+2 t\left(\langle\bar{z}, y\rangle_{\mathscr{X}}+\lambda\langle\bar{y}, y\rangle_{\mathscr{X}}\right)+t^{2}\left\langle\left(\boldsymbol{M}_{\mu}+\psi+\lambda\right) y, y\right\rangle_{\mathscr{X}}
\end{aligned}
$$

with $\lambda$ as above. The minimisation property of $\bar{y}$ ensures that $\rho(t)$ is minimised at 0 for any $y \in \mathscr{F}_{*}$ with $y \perp \bar{y}$. Thus, $\langle\bar{z}, y\rangle_{\mathscr{X}}=0$ for such $y$. Since $z$ is orthogonal (in the sense of the $y_{-} Y^{*}$ duality corresponding to the $\mathscr{X}$ inner product) to everything in $\mathscr{Y}_{*}$ which is orthogonal to $\bar{y}$, i.e.

$$
\bar{z} \perp\left\{y \in \mathscr{Y}: y \perp \operatorname{sp}\left\{\hat{y}_{1}, \ldots, \hat{y}_{K^{\prime}}, \bar{y}\right\}\right\}
$$

we must have $\bar{z} \in \operatorname{sp}\left\{\hat{y}_{1}, \ldots, \hat{y}_{K^{\prime}}, \bar{y}\right\}$. Hence $\bar{z} \in \mathscr{Y} \subset \mathscr{D}$ so $\bar{y} \in \mathscr{D}_{\mu, \psi}$ and $\bar{z}=\boldsymbol{M}_{\mu, \psi} \bar{y}$. Also, for $k=1, \ldots, K^{\prime}$ we have

$$
\begin{aligned}
\left\langle\hat{y}_{k}, \bar{z}\right\rangle_{\mathscr{X}} & =\left\langle\hat{y}_{k}, \boldsymbol{M}_{\mu, \psi} \bar{y}\right\rangle_{\mathscr{X}} \\
& =\left\langle\boldsymbol{M}_{\mu, \psi} \hat{y}_{k}, \bar{y}\right\rangle_{\mathscr{X}}=\lambda_{k} \quad\left\langle\hat{y}_{k}, \bar{y}\right\rangle_{\mathscr{X}}=0
\end{aligned}
$$

where $\lambda_{k^{\prime}}\left(k^{\prime}<K\right)$ is the eigenvalue corresponding to $w_{k^{\prime}}=\hat{y}_{k} \bar{U}$. Hence, $\bar{z} \in \operatorname{sp}\{\bar{y}\}$, i.e. $\bar{z}=\tilde{\lambda} \bar{y}$ for some $\tilde{\lambda}$. Clearly $\tilde{\lambda}=\hat{\lambda} \leqslant \lambda_{K}$ and $\bar{u}:=\bar{y} \bar{U}$ is an eigenfunction of $\boldsymbol{A}_{\psi}$ with $\boldsymbol{A}_{\psi} \bar{u}=\left(\boldsymbol{M}_{\mu, \psi} \bar{y}\right) \bar{U}=\bar{z} \bar{U}=\bar{\lambda} \bar{u}$. The ordering (3.1), i.e. the definition of $\lambda_{K}$, then ensures $\tilde{\lambda} \geqslant \lambda_{K}$ so $\boldsymbol{A}_{\psi} \bar{u}=\lambda_{K} \bar{u}$. To within the arbitrariness in the specification of the eigenfunctions we can take $w_{K}=\bar{u}$. 
This argument is essentially the Courant minimax theorem (cf, e.g. [2]), adapted to the present definition of $\boldsymbol{A}_{\psi}$. The characterisation by (3.8) permits us, as in [1], to show the continuous dependence of each eigenvalue $\lambda_{k}=\lambda_{k}(\psi)$ (and of each corresponding eigenfunction $w_{k}$, to within the arbitrariness inherent in specification of the eigenfunctions) on $\psi$, topologised by the $P^{*}$ norm.

Theorem 11. Let $\psi=\psi_{i} \rightarrow \vec{\psi}$ strongly in $\mathscr{P}^{*}$ and assume there is a bounded sequence $\left\{\varphi=\varphi_{i}\right\}$ in $\left(\hat{y}_{\nu}\right)^{*}$ such that $\psi_{i} \geqslant \varphi_{i}$. Then as $i \rightarrow \infty$ one has

$$
\lambda_{k}=\lambda_{k, i}:=\lambda_{k}\left(\psi_{i}\right) \rightarrow \bar{\lambda}_{k}:=\lambda_{k}(\bar{\psi})
$$

for each $k=1,2, \ldots$ Correspondingly, we have

$$
w_{k}=w_{k, i}:=w_{k}\left(\psi_{i}\right) \rightarrow \bar{w}_{k}:=w_{k}(\bar{\psi}) \text { in } \mathscr{V}=\mathscr{H}^{\prime}(\mathbf{\Omega})
$$

to within the arbitrariness inherently associated with our specification of the eigenfunctions.

Proof. The argument is essentially the same as the corresponding argument in [1], inductively using the variational characterisation:

$$
\begin{aligned}
& \lambda_{K}(\psi)=\min \left\{\langle(A+\psi) u, u\rangle: u \in \cup_{\mu} \mathscr{Y}_{\mu, K}(\psi)\right\} \\
& \mathscr{S}_{\mu, K}(\psi):=\left\{u=y \bar{U}: y \in Y \text { with }|y|_{\mathscr{Q}}=1 ; \bar{U}=\bar{U}_{\mu, j} ; u \perp w_{k}(\psi) \text { for } k<K\right\} \\
& w_{k}(\psi)=\arg \min \left\{\langle(A+\psi) w, w\rangle: w \in \cup_{\mu} \mathscr{Y}_{\mu, k}(\psi)\right\}
\end{aligned}
$$

given by lemma 10. The inductive hypothesis is to assume the result (3.10), (3.11) known for $k<K$ and fix $K$. We now write $\lambda=\lambda_{i}, w=w_{i}, \bar{\lambda}$, and $\bar{w}$ for $\lambda_{K}=\lambda_{K, i}$, $w_{K}=w_{K, i}, \bar{\lambda}_{K}$, and $\bar{w}_{K}$.

We first wish to show that $\lim \sup \lambda_{i} \leqslant \bar{\lambda}$. To this end, obtain $\tilde{u}=\tilde{u}_{i}$ by applying the Gram-Schmidt procedure to $\left\{w_{1, i}, \ldots, w_{K-1, i}, \bar{w}\right\}$ so

$$
\tilde{u}=N\left(\bar{w}-\sum_{k<K} C_{k} w_{k}\right)
$$

where $N=N_{i}$ is a normalising constant and, noting the orthonormality of $\left\{w_{k}=w_{k, i}\right.$ : $k<K\}$, we have $C_{k}=C_{k, i}=\left\langle w_{k}, \bar{w}\right\rangle$. Since, by the inductive hypothesis, $w_{k} \rightarrow \bar{w}_{k}$ and $\bar{w}_{k} \perp \bar{w}$ for $k<K$, we have

$$
N=N_{i} \rightarrow 1 \quad C_{k}=C_{k, i} \rightarrow 0 \text { for } k<K .
$$

From (3.13), (3.14) it follows that $\bar{u} \rightarrow \bar{w}$ in $\mathscr{V}$. Actually, we know that $\bar{w}$ has the form $\bar{y} \tilde{U}$ with $\bar{U}=\bar{U}_{\mu, j}$ (some fixed $\mu, j$ ) and $\bar{y} \in \mathcal{Y}$ with $|\bar{y}|_{x}=1$ and $\boldsymbol{M}_{\mu, \bar{\psi}} \bar{y}=\bar{\lambda} \bar{y}$. In (3.13) we have $C_{k}=0$ for any $w_{k}$ not corresponding to the same $(\mu, j)$ so we can set

$$
\tilde{y}:=N\left(\tilde{y}-\sum_{k<K} C_{k} y_{k}\right)
$$

where $w_{k}=y_{k} \bar{U}_{\mu, j}($ any $\mu, j)$ and have $\tilde{u}=\tilde{y} \breve{U}$ (same $\tilde{U}$ as for $\bar{w}$ ). We have $\tilde{y}=\tilde{y}_{i} \rightarrow \bar{y}$ in $\mathscr{y}$ whence also $\left\{\tilde{y}_{i}^{2}\right\}$ is convergent in $\mathscr{P}$. Now lemma 10 gives

$$
\begin{aligned}
\lambda-\lambda_{K . i} & \leqslant\langle(\boldsymbol{A}+\psi) \tilde{u}, \tilde{u}\rangle \\
& =\left\langle\left(\boldsymbol{M}_{\mu}+\bar{\psi}\right) \tilde{y}, \tilde{y}\right\rangle_{\mathscr{x}}+\left\langle\psi-\bar{\psi}, \tilde{y}^{2}\right\rangle_{\mathscr{x}} .
\end{aligned}
$$


We have

$$
\left\langle\left(\boldsymbol{M}_{\mu}+\bar{\psi}\right) \tilde{y}, \tilde{y}\right\rangle \rightarrow\left\langle\left(\boldsymbol{M}_{\mu}+\bar{\psi}\right) \bar{y}, \bar{y}\right\rangle=\bar{\lambda}
$$

as $\tilde{y} \rightarrow \bar{y}$ in $y$ and

$$
\left\langle\psi-\bar{\psi}, \tilde{y}^{2}\right\rangle \rightarrow 0
$$

as $\tilde{y}^{2} \rightarrow \bar{y}^{2}$ in $\mathscr{P}$ and $\psi \rightarrow \bar{\psi}$ in $\mathscr{P}^{*}$ (even weak convergence would suffice). Thus, lim sup $\lambda \leqslant \bar{\lambda}$.

We now wish to show, conversely, that $\lim \inf \lambda_{K, i} \geqslant \tilde{\lambda}$, giving $(3.10)$, and that (3.11) holds. Each $w=w_{K, i}$ has the form $y \bar{U}$ by our specifications and, as for lemma 10 , the upper bound on $\lambda$ which we have just obtained restricts attention to $\bar{U}=\bar{U}_{\mu, j}$ for a finite set of relevant $(\mu, j)$. Thus, possibly subdividing $\left\{w_{K, i}\right\}$ into alternative $\dagger$ subsequences, we may assume a fixed $\bar{U}$ and that this $\bar{U}$ is to be used in specifying $\bar{w}$. For each $w=w_{K, i}$, then we have $w=y \tilde{U}$ with $y \in \mathscr{O},|y|_{\mathscr{X}}=1$, and $\boldsymbol{M}_{\mu, \psi} y=\lambda y$. The same estimate (3.9) as in lemma 10 (recalling the assumed boundedness of $\left\{\varphi=\varphi_{i}\right\}$ ) bounds $\left\{y=y_{i}\right\}$ in $o y$ so we may assume (again possibly taking a subsequence) that $\left\{y_{i}\right\}$ converges (weakly in $\mathscr{Y}$ so strongly in $\mathscr{X}$ ) to some $\bar{y} \in \mathscr{Y}$ with $|\bar{y}|_{\mathscr{X}}=1$. For $k<K$ we need consider only $\left\{\hat{y}_{1}, \ldots, \hat{y}_{K^{\prime}}\right\}=\left\{y: w_{k}=y \bar{U}\right.$ with $\left.k<K\right\}$ as earlier, except that $\hat{y}_{j}=\hat{y}_{j, t}$ now (but we are considering the fixed $\tilde{U}$ as for $w, \bar{w}$ ) and, similarly, $\bar{y}_{j}:=\hat{y}_{j}(\bar{\psi})$; note that the corresponding indices are independent of $i$ by our inductive assumption. Then

$$
\left\langle y, \bar{y}_{j}\right\rangle_{\mathscr{X}}=\left\langle y, \bar{y}_{j}-\hat{y}_{j}\right\rangle_{\grave{x}} \rightarrow 0
$$

since $\left\langle y, \hat{y}_{j}\right\rangle_{\mathscr{X}}=0$ and, inductively, (3.11) giving $w_{k}=\hat{y}_{j} \rightarrow \bar{w}_{k}=\bar{y}_{j}$ in $\mathscr{H}$ corresponds to $\left|\bar{y}_{j}-\hat{y}_{j}\right|_{\mathscr{E}} \rightarrow 0$. Hence, in the limit $\left\langle\bar{y}, \bar{y}_{j}\right\rangle_{\mathscr{X}}=0$ and $\left\langle\bar{y} \bar{U}, w_{k}\right\rangle_{\mathscr{H}}=0$ for each $k<K$. We also have

$$
\left\langle\psi-\bar{\psi}, y^{2}\right\rangle \rightarrow 0
$$

since we have assumed $\psi \rightarrow \bar{\psi}$ strongly $\ddagger$ in $\mathscr{P}^{*}$ and $\left\{y^{2}\right\}$ is bounded in $\mathscr{P}$ by lemma 5 . Now choose $\tilde{\lambda}$ large enough that $\left(\boldsymbol{M}_{\mu}+\bar{\psi}+\bar{\lambda}\right): \mathscr{Y} \rightarrow \mathrm{Y}^{*}$ is (strictly) monotonic so the functional: $y \mapsto\left\langle\left(\boldsymbol{M}_{\mu}+\bar{\psi}+\bar{\lambda}\right) y, y\right\rangle$ is (strictly) convex on $y$ and so lower semicontinuous with respect to the weak topology of $y$. We have

$$
\lambda=\left\langle\left(\boldsymbol{M}_{\mu}+\bar{\psi}+\tilde{\lambda}\right) y, y\right\rangle_{\mathscr{E}}-\tilde{\lambda}+\left\langle\psi-\bar{\psi}, y^{2}\right\rangle_{\mathscr{X}}
$$

so, as $y-\bar{y}$ in $y$, we have

$$
\begin{aligned}
\lim \inf \lambda & \geqslant\left\langle\left(M_{\mu}+\bar{\psi}+\tilde{\lambda}\right) \bar{y}, \bar{y}\right\rangle-\tilde{\lambda} \\
& =\left\langle\left(\boldsymbol{M}_{\mu}+\bar{\psi}\right) \bar{y}, \bar{y}\right\rangle_{\mathscr{X}}=\langle(\boldsymbol{A}+\psi)(\bar{y} \bar{U}), \bar{y} \bar{U}\rangle_{\mathscr{H}} \\
& \geqslant \min \left\{\langle(\boldsymbol{A}+\bar{\psi}) u, u\rangle: u \in \cup_{\mu} \mathscr{S}_{\mu, K}\right\}=\bar{\lambda}_{K} .
\end{aligned}
$$

This shows that $\lambda=\lambda_{K}\left(\psi_{i}\right) \rightarrow \bar{\lambda}_{K}$ along subsequences for which $w_{K, i}=y_{i} \bar{U}$ with $\bar{U}$ fixed and $\left\{y_{i}\right\}$ weakly convergent in 9 . The uniqueness of the limit shows $\lambda_{K, i} \rightarrow \bar{\lambda}_{K}$ along the entire original sequence, proving (this step of the induction for) (3.10).

\footnotetext{
$\dagger$ These alternatives would correspond to equally valid ways of specifying $\bar{w}_{K}$, as is shown by the subsequent argument.

$\ddagger$ This corrects a minor error in [1] where, at the corresponding point, only weak convergence $\psi_{i}-\psi$ in $\mathscr{P}^{*}$ was assumed-which seems inadequate if one has only weak convergence: $y_{i}-\bar{y}$ in $\mathscr{y}$. Note that we need only $\lim \sup \left\langle\psi_{i}-\bar{\psi}, y^{2}\right\rangle \leqslant 0$ so weak convergence would be adequate if supplemented by a one-sided bound: $\psi \leqslant \bar{\psi}+\theta_{j}$ with strong convergence: $\theta_{i} \rightarrow 0$ in $\mathscr{P}^{*}$. It remains open as to whether weak convergence could suffice in general.
} 
As in lemma 10, we now have $\bar{y} \in \mathscr{D}_{\mu, \bar{\psi}}$ and that $\bar{y} \bar{U}$ is an eigenfunction of $\boldsymbol{A}_{\psi}$ corresponding to the eigenvalue $\lambda_{K}$ so we could set $\dagger \bar{w}_{K}:=\bar{y} \vec{U}$ in constructing the sequence of eigenpairs recursively. Note that

$$
y \mapsto\left\langle\left(\boldsymbol{M}_{\mu}+\bar{\psi}+\bar{\lambda}\right) y, y\right\rangle_{\bar{x}}^{1 / 2}=:\|y\|_{\bar{\psi}}
$$

is an equivalent Hilbert space norm for $y$ and we have just shown that

$$
\left\|y_{i}\right\|_{\psi}=\left(\lambda_{K, i}+\tilde{\lambda}\right)^{1 / 2} \rightarrow\left(\bar{\lambda}_{K}+\tilde{\lambda}\right)^{1 / 2}=:\|y\|_{\bar{\psi}}
$$

which, with the weak convergence $\left(y_{i} \rightarrow \bar{y}\right.$ in $\left.y\right)$ implies norm convergence: $\left\|y_{i}-\bar{y}\right\|_{\psi} \rightarrow 0$, i.e. $y_{i} \rightarrow \bar{y}$ strongly in $y$. Thus, $y_{i} \rightarrow \bar{y}$ in $\vartheta_{0}$ and $w_{K . i}=y_{i} \bar{U} \rightarrow \bar{y} \bar{U}=: \bar{w}_{K}$ in $\mathscr{V}=y_{0} \otimes U$, as desired $t$.

This completes the inductive step and, since the inductive hypothesis is vacuous for $K=1$, the proof of the theorem is complete by induction on $K$.

\section{The approximation scheme}

The method of generalised interpolation [3,4] is a quite general approach to the approximate solution of ill-posed problems. Typically, one must first observe the equivalence of the problem to specification of the values for a sequence of functionals $\left\{\lambda_{k}(\cdot)\right\}$ but here, as in [1], the nature of the problem already presents it in this form.

The simplest version of the method is the procedure $\left(\boldsymbol{P}_{N}\right)$ described in $\S 1$. The relevant hypotheses $\S$ are as follows.

The norm $\|\cdot\|_{*}$ (determining a reflexive Banach space $\mathscr{P}_{*}$ ) topologising the relevant potentials is such that if $\psi_{\nu} \rightarrow \bar{\psi}$ weakly in $\mathscr{P}_{*}$ with $\left\|\psi_{v}\right\|_{*} \rightarrow\|\bar{\psi}\|_{*}$, then $\psi_{v} \rightarrow \bar{\psi}$ strongly in $\mathscr{P}_{*}$.

Weak convergence $\psi_{v} \rightarrow \bar{\psi}$ in $\mathscr{P}_{*}$ implies $\lambda_{k}\left(\psi_{v}\right) \rightarrow \lambda_{k}(\bar{\psi})$ for each $k=1,2, \ldots$

The constraint set $\Psi_{*} \subset \mathscr{P}_{*}$ is such that the problem $\lambda_{k}(\psi)=\bar{\lambda}_{k}$ for $k=1,2, \ldots$ with $\psi \in \Psi_{*}$ has at most one (minimum norm) solution $\bar{\psi}$.

\footnotetext{
$\dagger$ This is not unique but is within the inherent arbitrariness associated with our specification of eigenfunctions, especially when $\bar{\lambda}_{K}$ is not simple.

\$ Again, this is only along the subsequences for which one already has weak convergence $y_{i} \rightarrow \bar{y}$ in $\partial$ and $\bar{U}$ fixed. This time, however, the limit is not unique. If $\bar{\lambda}_{K}$ were simple (not counting the multiplicities induced by multiplicity of $\mu$ in $\sigma(S)$ which are easy to handle), then one could just reorient $w=w_{K, i}$ as necessary-e.g. replacing $w$ by $-w$ if, otherwise, one had $\left\langle w, \bar{w}_{K}\right\rangle<0$ - to ensure $w \rightarrow \bar{w}$ for the full sequence. In general, the 'correct' result when $\bar{\lambda}_{K-1}<\bar{\lambda}_{K}=\ldots=\bar{\lambda}_{L}<\bar{\lambda}_{L+1}$ is to let $\boldsymbol{P}_{i}$ be the $\mathscr{H}$-orthogonal projection on $\operatorname{sp}\left\{w_{K}, \ldots, w_{L}\right\}$ and observe that this converges to $\overline{\boldsymbol{P}}$ in the operator norm for $\mathscr{V}$.

In any case, our application of this theorem will only use (3.10). One needs (3.11) inductively for the argument but it is adequate to extract the subsequence repeatedly and then to rely on the uniqueness of the eigenvalue sequence (3.1) to have (3.10) for the full original sequence.

$\S$ The property (4.1) is referred to as the 'Efimov-Stečkin property'. We refer to (4.2), (4.3) briefly as '(weak) continuity' and 'uniqueness', respectively.
} 
Under these hypotheses (4.1)-(4.3) it is a general result [6] that

$$
\psi_{N} \rightarrow \bar{\psi} \quad \text { (strongly in } \mathscr{P}_{*} \text { ) as } N \rightarrow \infty
$$

where we assume the data $\left\{\bar{\lambda}_{k}\right\}$ are consistent (i.e. a solution exists in (4.3)) and, as for $\left(P_{N}\right)$, each $\psi_{N}(N=1,2, \ldots)$ is defined as the minimum norm element of $\Psi_{*}$ subject to matching the given values $\bar{\lambda}_{k}$ of $\lambda_{k}(\cdot)$ for $k=1, \ldots, N$.

Rather than prove the result in this form, we turn instead to consideration of a more general version which permits the use of (implementable) approximate procedures for the ' $N$ th stage' computations. Before doing this we comment on the hypotheses.

It is known that (4.1) holds for any uniformly convex Banach space, in particular for Hilbert spaces. Our major effort, to this point, has been to show that one obtains continuity of the eigenvalues, viewed as nonlinear functionals on the (radial) potential $\psi$, using anorm convergence in the specific space $\mathscr{P}^{*}$ and subject to a lower bound condition. Our first observation is that (4.1) and (4.2) need only hold on the constraint set $\Psi_{*}$. We will assume†:

the constraint set $\Psi_{*}$ is in $\mathscr{P}^{*}$ and for each $\mathscr{P}_{*}$-bounded subset $\Psi_{0} \subset \Psi_{*}$, there exists a suitable $v$ and a number $m$ such that each $\psi \in \Psi_{0}$ has a lower bound $\varphi \in\left(\hat{Y}_{v}\right)^{*}$ with $\psi \geqslant \varphi,\|\varphi\| \leqslant m\left(\left(\hat{\vartheta}_{v}\right)^{*}\right.$ norm $)$

and obtain (the restricted form of) the condition (4.2) by requiring compact embedding: $\mathscr{P}_{*} \rightarrow \mathscr{P}^{*}$. We would like to permit consideration of potentials $\psi$ involving (radial) measures and note that our efforts in working with such a weak space as $\mathscr{P}^{*}$ do, indeed, have the value of permitting this, even after the norm is strengthened (defining $\mathscr{P}_{*}$ ) to have this compact embedding. Note that we are not assuming that $\Psi_{*}$ itself is compact $\ddagger$ in $\mathscr{P}^{*}$. but only a relative pre-compactness in $\Psi_{*}$ of sets bounded with respect to $\mathscr{P}_{*}$ norm without having to specify any particular such $\mathscr{P}_{*}$ bound in specifying $\Psi_{*}^{*}$

The uniqueness property (4.3) is, at present, terra incognita for EVP, even for the case of radial potentials. In the one-dimensional case $\left(\Omega:=(-1,1) \subset \mathbb{R}^{1}\right)$ radiality just means that the potential is known to be symmetric on the interval and that is known to ensure uniqueness [7]. This suggests the possibility that (4.3) may hold $\$$ for quite

$\dagger$ The simplest form of this, of course, would be to have $\psi \geqslant 0$ for $\psi \in \Psi_{*}$ or, slightly more generally, a one-sided condition that $\psi \geqslant \boldsymbol{C}$ for a constant $\boldsymbol{C}$ depending only on the $\mathscr{P}^{*}$ norm of $\psi$.

$\ddagger$ This assumption (corresponding, e.g., to an assumed a priori bound on $\Psi_{*}$ in a space as $\mathscr{P}_{*}$ ) would permit a simpler approach. The map

$$
\Lambda: \psi \mapsto\left[\lambda_{1}(\psi), \ldots\right]: \Psi_{*} \leftarrow \mathbb{R}^{x}
$$

(taking $\mathbb{R}^{\infty}$ with the product topology) would be a continuous injective map from a compact Hausdorff space. By a standard result of point-set topology, $\Lambda$ would then have compact range and a uniformly continuous inverse. The (uniform) continuity of the inverse would mean that, in specifying $\Psi$, we have assumed away the ill-posedness of the inverse problem EVP. The difficulty lies in justification of any specific a priori bound on the potential $\bar{\psi}$.

$\S$ An interesting stronger conjecture is that one might be able to recover $\psi$ from knowledge only of those eigenvalues of $\boldsymbol{A}_{\psi}$ associated with purely radial eigenfunctions, i.e. from $\sigma\left(\boldsymbol{M}_{0, \psi}\right)$. This seems unlikely, however, as in the one-dimensional case it would correspond to knowing $\psi$ symmetric but only giving alternate eigenvalues-those with even eigenfunctions. More plausible would be to conjecture, for example, that $\sigma\left(\boldsymbol{M}_{0, \psi}\right)$ together with one other spectrum-i.e. $\sigma\left(\boldsymbol{M}_{\mu, \psi}\right)$ for some other $\mu \in \sigma(\boldsymbol{S})$-would suffice for uniqueness. 
general $\Psi_{*} \subset \mathscr{P}^{*}$, satisfying (4.5), but this remains entirely conjectural at present. Here we take the uniqueness condition (4.3) as an a priori hypothesis without investigating specific settings (i.e. more concrete conditions) permitting its direct verification.

We turn now to the more general approximation procedure, relaxing $\left(\boldsymbol{P}_{N}\right)$ somewhat. For this we assume that we are given $\delta_{N}>0$ and positive functions $\varepsilon_{N, k}(\psi)>0$ for $k \in N$ and $\psi \in \Psi_{*}$. The procedure (at this ' $N$ th stage') is then

$$
\begin{aligned}
\left(\boldsymbol{P}_{\mathrm{a}, N}\right) \quad & \text { Let } \Psi_{N}:=\left\{\psi \in \Psi_{*}:\left|\lambda_{k}(\psi)-\bar{\lambda}_{k}\right| \leqslant \varepsilon_{N, k}(\psi)\right. \text { for } \\
& k=1, \ldots, N\} \text { and select } \psi_{N} \in \Psi_{N} \text { such that }\left\|\psi_{N}\right\|_{*} \\
& \leqslant \inf \left\{\|\psi\|_{*}: \psi \in \Psi_{N}\right\}+\delta_{N} .
\end{aligned}
$$

We note that $\left(\boldsymbol{P}_{\mathrm{a}, N}\right)$ does not determine $\psi_{N}$ uniquely.

It is important to realise, at this point, that this approach implicitly addresses the difficulties normally associated with noisy correlated (redundant) data; compare, e.g., $[6,8]$. We envision, here, a sequence of (measured) data sets $\left\{\hat{\lambda}_{k}\right\}_{N}$ of the following nature: (i) while each set may include only finitely many $k$, all the (necessary) eigenvalue measurements become eventually included and (ii) each individual eigenvalue measurement (i.e. fixed $k$ ) becomes arbitrarily accurate as $N \rightarrow \infty$. Thus, with no loss of generality we may assume $\left\{\hat{\lambda}_{k}\right\}_{N}=\left\{\hat{\lambda}_{N, k}: k=1, \ldots, N\right\}$ and that we have available accuracy estimates $\hat{\varepsilon}_{N, k}>0$ bounding each measurement error: $\left|\hat{\lambda}_{N, k}-\bar{\lambda}_{k}\right| \leqslant$ $\hat{\varepsilon}_{N, k}(k=1, \ldots, N ; N=1,2, \ldots)$ with $\hat{\varepsilon}_{N, k} \rightarrow 0$ as $N \rightarrow \infty$ for each fixed $k$. Now choose numbers $\varepsilon_{N, k}>\hat{\varepsilon}_{N, k}, \delta_{N}>0$ in such a way that $\varepsilon_{N, k} \rightarrow 0, \delta_{N} \rightarrow 0$. Then, regardless of the nature of any redundancies or consistency requirements of the problem (which will necessarily be satisfied by the true, exact data set $\left.\left\{\bar{\lambda}_{k}=\lambda_{k}(\bar{\psi})\right\}\right)$ the set $\Psi_{N}$ defined in $\left(\boldsymbol{P}_{\mathrm{a}, N}\right)$ must always be non-empty (since, by construction, we will always have, e.g., $\bar{\psi} \in \Psi_{N}$ for each $N$-although this does not give $\psi_{N}=\bar{\psi}$ or even force $\psi_{N}$ to be close to $\bar{\psi}$ for any particular $N)$. Thus, implementation of our computational procedure $\left(\boldsymbol{P}_{\mathrm{a}, N}\right)$ is always feasible (by the definition of 'inf') and the conclusion of the theorem is thathowever $\left(\boldsymbol{P}_{\mathrm{a}, N}\right)$ may be implemented-the approximating sequence $\left\{\psi_{N}\right\}$ always converges to the correct $\bar{\psi}$ (in the sense of the norm used for the minimisation). There is thus no need, when employing this approach, to make a preliminary data reduction to a 'minimal' (independent) set.

Theorem 12. Let $\mathscr{P}^{*}$ be as in theorem 11 and let $\mathscr{P}_{*}$ be a reflexive Banach space (with norm $\|\cdot\|_{*}$ ) embedding compactly in $\mathscr{P}^{*}$ and satisfying (4.1). Let $\Psi_{*}$ be a closed convex $\dagger$ subset of $\mathscr{P}_{*} \subset \mathscr{P}^{*}$ satisfying (4.5). Assume $0<\delta_{N} \rightarrow 0$ and $0<\varepsilon_{N, k}(\cdot) \rightarrow 0$ for each fixed $k$, uniformly on $\mathscr{P}_{*}$-bounded subsets of $\Psi_{*}$. The operator $\boldsymbol{A}_{\psi}$ is defined (as in $\S 2$ ) for radial potentials $\psi \in \Psi_{*}$ and the spectrum $\left[\lambda_{k}(\psi): k=1,2, \ldots\right]=\sigma\left(\boldsymbol{A}_{\psi}\right)$ is as in (3.1), in increasing order with multiplicities. Suppose, for $k=1,2, \ldots$, we have $\bar{\lambda}_{k}=\lambda_{k}(\bar{\psi})$ for some unique $\neq \bar{\psi} \in \Psi_{*}$. Then (4.4) holds for any sequence $\left\{\psi_{N}\right\}$ in $\Psi_{*}$ obtained by the procedure $\left(\boldsymbol{P}_{\mathrm{a}, N}\right)$ for $N=1,2, \ldots$.

Proof. The first observation is that $\bar{\psi} \in \Psi_{N}$ so

$$
\left\|\psi_{N}\right\|_{*} \leqslant\|\ddot{\psi}\|_{*}+\delta_{N} \quad \lim \sup \left\|\psi_{N}\right\|_{*} \leqslant\|\bar{\psi}\|_{*} .
$$

$\dagger$ It is sufficient that $\Psi_{*}$ be closed in the weak topology of $\mathscr{P}_{*}$.

$\ddagger$ It is sufficient that $\bar{\psi}$ be unique among minimum norm solutions. The general condition (4.3) asserts that for any eigenvalue sequence $\left(\tilde{\lambda}_{k}\right)$ which can arise (consistency) the minimum norm solution in $\Psi_{*}$ is unique as asserted. 
We may then assume (extracting a subsequence if necessary) $\psi_{N} \rightarrow \tilde{\psi}$ : weak convergence in $\mathscr{P}_{*}$ for some $\tilde{\psi}$. As we assumed $\Psi_{*}$ closed and convex, it follows that $\tilde{\psi} \in \Psi_{*}$ and we will show that $\tilde{\psi}$ is a (minimum norm) solution of

$$
\lambda_{k}(\psi)=\bar{\lambda}_{k} \quad \text { for } k=1,2, \ldots
$$

whence $\tilde{\psi}=\bar{\psi}$ by the assumed uniqueness. The uniqueness of the limit shows that the possible extraction of a subsequence above was nugatory: one has $\psi_{N} \rightarrow \bar{\psi}$ for the full sequence $\left\{\psi_{N}\right\}$.

To see (4.7) for $\tilde{\psi}$, note that boundedness of $\left\{\psi_{N}\right\}$ in $\mathscr{P}_{*}$ gives $\varepsilon_{N, k}:=\varepsilon_{N, k}\left(\psi_{N}\right) \rightarrow 0$ so $\lambda_{k}\left(\psi_{N}\right) \rightarrow \bar{\lambda}_{k}$ by $\left(P_{\mathrm{a}, N}\right)$-considering only $N \geqslant k$, of course, for each $k=1,2, \ldots$ On the other hand, the assumed compactness of the embedding: $\mathscr{P}_{*} \rightarrow \mathscr{P}^{*}$ means that weak convergence: $\psi_{N} \quad \tilde{\psi}$ in $\mathscr{P}_{*}$ implies strong convergence: $\psi_{N} \rightarrow \tilde{\psi}$ in $\mathscr{P}^{*}$. The condition (4.5), with boundedness in $\mathscr{P}_{*}$ of $\left\{\psi_{N}\right\}$ also gives the 'lower bound condition' $\left(\psi_{N} \geqslant \varphi_{N}\right)$ of theorem 11 , so theorem 11 applies to give $\dagger \lambda_{k}\left(\psi_{N}\right) \rightarrow \lambda_{k}(\tilde{\psi})$ for each $k$ whence $\lambda_{k}(\tilde{\psi})=\bar{\lambda}_{k}$.

At this point we have weak convergence $\psi_{N} \rightarrow \tilde{\psi}$ in $\mathscr{P}_{*}$ (along the subsequence). The convexity of the norm gives lower semicontinuity with respect to the weak topology so $\psi_{N} \longrightarrow \tilde{\psi}$ implies

$$
\|\tilde{\psi}\|_{*} \leqslant \liminf \left\|\psi_{N}\right\|_{*} \leqslant\|\bar{\psi}\|_{*} .
$$

Since $\bar{\psi}$ is a minimum norm solution of (4.7) by assumption, the solution $\bar{\psi}$ cannot have smaller norm; hence $\|\tilde{\psi}\|_{*}=\|\bar{\psi}\|_{*}$ from (4.8) and $\tilde{\psi}$ is also a minimum norm solution. The assumed uniqueness of $\bar{\psi}$ then implies $\tilde{\psi}=\bar{\psi}$. As noted above, this gives weak convergence $\psi_{N} \rightarrow \bar{\psi}$ in $\mathscr{P}_{*}$ (along the full sequence) without yet using (4.1).

Now if we combine (4.6) with (4.8) we see that $\left\|\psi_{N}\right\|_{*} \rightarrow\|\bar{\psi}\|_{*}$. This, with the weak convergence, gives (4.4) subject to the assumption (4.1).

For implementation we note that one does not attempt to construct $\Psi_{N}$ and need not even construct $\psi_{N} \in \Psi_{N}$ directly as in $\left(P_{\mathrm{a}, N}\right)$. If one could produce any element $\tilde{\psi}_{N} \in \mathscr{P}_{*}$ for which one would have an estimate (for some $\psi_{N}$ as in $\left.\left(P_{\mathrm{a}, N}\right), N=1,2, \ldots\right)$ :

$$
\left\|\tilde{\psi}_{N}-\psi_{N}\right\|_{*} \leqslant \delta_{N}^{\prime}\left(\psi_{N}\right)
$$

with $\delta_{N}^{\prime}(\cdot) \rightarrow 0$ uniformly on $\mathscr{P}_{*}$-bounded sets in $\Psi_{*}$, then as an immediate corollary of theorem 12 , one also has $\tilde{\psi}_{N} \rightarrow \bar{\psi}$ in $\mathscr{P}_{*}$ as $N \rightarrow \infty$. We will not, however, attempt to reduce the proof of convergence of our computational implementation to theorem 12 , but instead will use an essentially similar argument to prove convergence directly.

Parametrised by $h>0$, we will need a family of computational approximations $\Psi_{*}(h)$ to $\Psi_{*}$ and an algorithm $\neq$ which takes $h, N$ and (a representation of) $\psi \in \Psi_{*}(h)$ as inputs and returns

$$
\Lambda_{N}(\psi ; h)=\left[\hat{\lambda}_{1}(\psi ; h), \ldots, \hat{\lambda}_{N}(\psi ; h)\right]
$$

$\dagger$ For present purposes (3.10) suffices: (3.11) is relevant only as part of the inductive argument for theorem 11.

$\ddagger$ One could attempt a finite-element discretisation of $(\boldsymbol{A}+\psi)$ from (2.11), using a finite-element subspace corresponding to a mesh parameter $h$, if $\psi$ were moderately smooth (or first approximate $\psi$ by a smoother $\bar{\psi})$. In view of the analysis above, one might more plausibly use such finite-element discretisations to $\left(\boldsymbol{M}_{\mu+\psi}\right)$ for relevant $\mu$, assuming $\sigma(S)$ accurately known. This effectively produces (sparse) $n(h) \times n(h)$ symmetric matrices whose 'first' $N$ eigenvalues could be computed and taken as giving $\Lambda_{N}(\psi ; h)$. In general, such a procedure would give $\hat{\lambda}_{k}(\psi ; h) \rightarrow \lambda_{k}(\psi)$ as $h \rightarrow 0$. 
approximating $\left[\lambda_{1}(\psi), \ldots, \psi_{N}(\psi)\right]$. Reasonable properties of such a computational procedure would be:

$$
\begin{aligned}
& \text { given any } \psi \in \Psi_{*} \text { there exists } \tilde{\psi} \in \Psi_{*}(h) \text { with }\|\psi-\tilde{\psi}\|_{*} \leqslant \\
& \left.\delta(\psi ; h) \text { and given any } \tilde{\psi} \in \Psi_{*} h\right) \text { there exists } \psi \in \Psi_{*} \text { with } \\
& \|\psi-\tilde{\psi}\|_{*} \leqslant \delta(\tilde{\psi} ; h) \text { where } \delta(\cdot ; h) \rightarrow 0 \text { as } h \rightarrow 0 \text { uniformly on } \\
& \mathscr{P}_{*} \text {-bounded sets; we may also assume }(4.5) \text { for } \Psi_{*}(h), \\
& \text { uniformly in } h \text { for small } h ; \\
& \left|\bar{\lambda}_{k}(\psi ; h)-\lambda_{k}(\psi)\right| \rightarrow 0 \text { as } h \rightarrow 0 \text { for each fixed } k=1,2, \ldots, \\
& \text { uniformly on } \mathscr{P}_{*} \text {-bounded sets. }
\end{aligned}
$$

Without further concern for the details of possible construction of such algorithms, we indicate how the availability of a computational implementation satisfying (4.10) and (4.11) could be used to obtain a computable sequence $\left\{\tilde{\psi}_{N}\right\}$ converging in $\mathscr{P}_{*}$ norm to $\bar{\psi}$.

We wish to replace the approximation procedure $\left(\boldsymbol{P}_{\mathrm{a}, N}\right)$ by a more explicitly implementable computational procedure:

$$
\begin{aligned}
\left(\boldsymbol{P}_{\mathrm{c}, N}\right) \quad \text { choose } h=h_{N} \text { small enough that } \Psi_{N}(h) \\
:=\left\{\psi \in \Psi_{*}(h):\left|\hat{\lambda}_{k}(\psi ; h)-\tilde{\lambda}_{k}\right| \leqslant \tilde{\varepsilon}_{N, k} \text { for } k \leqslant N\right\} \\
\text { is non-empty and select } \tilde{\psi}_{N} \in \tilde{\psi}_{N}:=\Psi_{N}\left(h_{N}\right) \text { such that } \\
\left\|\tilde{\psi}_{N}\right\|_{*} \leqslant \tilde{v}_{N}+\tilde{\delta}_{N} \text { where } \tilde{v}_{N}:=\inf \left\{\|\tilde{\psi}\|_{*}: \tilde{\psi} \in \tilde{\Psi}_{N}\right\} .
\end{aligned}
$$

The actual computation involved in $\left(\boldsymbol{P}_{\mathrm{c}, N}\right)$ would be the use of some (standard) algorithm for nonlinear constrained optimisation to minimise $\|\tilde{\psi}\|_{*}$ (using a stopping criterion giving approximate minimisation to within $\tilde{\delta}_{N}>0$ of the infimum $\tilde{v}_{N}$ ) subject to the constraint: $\tilde{\psi} \in \tilde{\Psi}_{N}$. The computational difficulty of this will depend on the nature of $\|\cdot\|_{*}$, on the sizes of $\varepsilon_{N, k}$, on the size of $h_{N}$ and the computational difficulty in implementing $\Lambda_{N}\left(\cdot ; h_{N}\right)$, etc.

Theorem 13. Let $\boldsymbol{A}, \mathscr{P}^{*}, \mathscr{P}_{*}, \Psi_{*},\left\{\bar{\lambda}_{k}\right\}$ be as for theorem 12 and assume implementable computational approximations $\Psi_{*}(h), \Lambda_{N}(\cdot ; h)$ are available (for small $h>0$ ) satisfying (4.10), (4.11). Assume $0<\tilde{\delta}_{N} \rightarrow 0$ and $\tilde{\varepsilon}_{N, k} \rightarrow 0$ for $k=1,2, \ldots$ as $N \rightarrow \infty$. Then, for each $N=1,2, \ldots$, one can choose $h=h_{N}$ so $\Psi_{N}:=\Psi_{N}\left(h_{N}\right)$ is non-empty (further requiring that $\left.h_{N} \rightarrow 0\right)$ and select $\tilde{\psi}_{N} \in \tilde{\Psi}_{N}$ as in $\left(P_{c, N}\right)$. For any such computed sequence $\left\{\tilde{\psi}_{N}\right\}$ we have

$$
\tilde{\psi}_{N} \rightarrow \bar{\psi} \quad \text { in } \mathscr{P}_{*} \text { norm } \quad \text { as } N \rightarrow \infty
$$

where $\bar{\psi}$ is given by (4.3).

Proof. Since $\bar{\psi} \in \Psi_{*}$ one has, by $(4.10)$, existence of $\bar{\psi}(h) \in \Psi_{*}(h)$ with $\|\bar{\psi}-\bar{\psi}(h)\|_{*} \leqslant$ $\tilde{\delta}(\tilde{\psi} ; h) \rightarrow 0$. This makes $\left\{\bar{\psi}(h): 0<h \leqslant h_{0}\right\}$ bounded so $(4.11)$ gives

$$
\left|\hat{\lambda}_{k}(\bar{\psi}(h) ; h)-\lambda_{k}(\bar{\psi}(h))\right| \leqslant \varepsilon_{N, k} / 2 \quad \text { for } k=1, \ldots, N
$$

for small enough $h$. On the other hand, $\bar{\psi}(h) \rightarrow \bar{\psi}$ in $\mathscr{P}_{*}$ (a fortiori in $\mathscr{P}^{*}$ ) and we have assumed (4.5) for $\Psi_{*}(h)$ so theorem 11 applies to give

$$
\left|\lambda_{k}(\bar{\psi}(h))-\bar{\lambda}_{k}\right| \leqslant \varepsilon_{N, k} / 2 \quad \text { for } k=1, \ldots, N .
$$


Combining these gives $\bar{\psi}(h) \in \Psi_{N}(h)$ for $h$ small enough so then $\Psi_{N}(h) \neq \emptyset$. Also requiring $h_{N} \leqslant h_{N}$ for any given sequence: $0<h_{N}^{\prime} \rightarrow 0$ lets us fix $\dagger h_{N}^{\prime}$. This fixes $\Psi_{N}:=\Psi_{N}\left(h_{N}\right)$ and we can find $\tilde{\psi}_{N} \in \Psi_{N}$, approximately minimising the norm, as in $\left(P_{\mathrm{c}, N}\right)$.

To show (4.12), we proceed as in the proof of theorem 12. Since $\bar{\psi}\left(h_{N}\right)$, as above, is in $\Psi_{N}$ for each $N$ and $\bar{\psi}\left(h_{N}\right) \rightarrow \bar{\psi}$, we see that

$$
\tilde{v}_{N} \leqslant\left\|\bar{\psi}\left(h_{N}\right)\right\|_{*} \rightarrow\|\bar{\psi}\|_{*}
$$

so $\left\{\tilde{v}_{N}\right\}$ is bounded and, as in (4.6), we have

$$
\lim \sup \left\|\tilde{\psi}_{N}\right\|_{*} \leqslant\|\tilde{\psi}\|_{*}
$$

so (extracting a subsequence if necessary) we may assume weak convergence: $\bar{\psi}_{N} \rightarrow \tilde{\psi}$ in $\mathscr{P}_{*}$. By (4.11) and the definition of $\tilde{\Psi}_{N}$ (noting that $\tilde{\varepsilon}_{N, k} \rightarrow 0$ ) we have $\lambda_{k}\left(\tilde{\psi}_{N}\right) \rightarrow \bar{\lambda}_{k}$ while theorem 11 gives $\lambda_{k}\left(\tilde{\psi}_{N}\right) \rightarrow \lambda_{k}(\tilde{\psi})$. It follows that $\tilde{\psi}$ is a solution of $(4.7)$ with $\|\tilde{\psi}\|_{*} \leqslant \liminf \left\|\bar{\psi}_{N}\right\| \leqslant\|\bar{\psi}\|_{*} ;$ the uniqueness property (4.3) then gives $\tilde{\psi}=\bar{\psi}$ and weak convergence $\tilde{\psi}_{N} \rightarrow \bar{\psi}$ for the full sequence. From (4.13) we also have $\left\|\tilde{\psi}_{N}\right\|_{*} \rightarrow\|\bar{\psi}\|_{*}$ so, by (4.1), we have (4.12) as asserted.

\section{Acknowledgments}

This paper is an outgrowth of [1] which was stimulated by conversations with $\mathrm{T}$ Suzuki. The stimulus to extend those results to the higher-dimensional case, specifically in a context of radial symmetry, came also from Suzuki and was further influenced by conversations with $\mathrm{U}$ Mosco and $\mathrm{J}$ McLaughlin. This research has been done during a visit to the Centre for Mathematical Analysis at the Australian National University; thanks are due to R S Anderssen and N Trudinger for the opportunity to make that visit and for the hospitality of the Centre. Support for the visit was provided by ANU and by the National Science Foundation under grant CDR-85-000108 through the Systems Research Center at the University of Maryland (UMCP). Additional support came as sabbatical leave from UMBC and under grant AFOSR-82-0271.

\section{References}

[1] Seidman T I 1985 A convergent approximation scheme for the inverse Sturm-Liouville problem Inverse Problems $1251-62$

[2] Weinberger H F 1974 Variational Methods for Eigenvalue Approximation (Philadelphia: SIAM)

[3] Seidman T I 1986 The method of 'generalized interpolation' for ill-posed problems Inverse Problems ed J R Cannon and U Hornung (Basel: Birkhäuser) pp 155-61

[4] Seidman T I 1981 Convergent approximation methods for ill-posed problems, Part I; general theory Control and Cybernetics 10 31-49

[5] Adams R A 1975 Sobolev Spaces (New York: Academic)

[6] Hald O H 1980 Inverse eigenvalue problems for the mantle Geophys. J. R. Astron. Soc. 62 41-8

[7] Gelfand I M and Levitan B M 1951 On the determination of a differential equation from its spectral function Izv. Akad. Nauk 15 (Engl. transl. Am. Math. Soc. 1 253-304)

[8] Sabatier P C 1978 Spectral and scattering inverse problems J. Math. Phys. 19 2410-25

$\uparrow$ Computationally, one might start with a trial $h_{n}=h_{N}^{\prime}$ and then, say, successively halve $h_{N}$ until one can obtain/compute some $\tilde{\psi} \in \Psi_{N}\left(h_{N}\right)$. 